\title{
On an Sir Epidemic Model for the COVID-19 Pandemic and the Logistic Equation
}

\author{
Manuel De la Sen ${ }^{1}{ }^{1}$ and Asier Ibeas ${ }^{2}$ \\ ${ }^{1}$ Institute of Research and Development of Processes IIDP, Department of Electricity and Electronics, \\ University of the Basque Country, Campus of Leioa, Leioa (Bizkaia), P.O. Box 48940, Spain \\ ${ }^{2}$ Department of Telecommunications and Systems Engineering, Universitat Autònoma de Barcelona, UAB, \\ 08193-Barcelona, Spain \\ Correspondence should be addressed to Manuel De la Sen; manuel.delasen@ehu.eus
}

Received 24 July 2020; Revised 10 September 2020; Accepted 18 November 2020; Published 9 December 2020

Academic Editor: Leonid Shaikhet

Copyright ( 92020 Manuel De la Sen and Asier Ibeas. This is an open access article distributed under the Creative Commons Attribution License, which permits unrestricted use, distribution, and reproduction in any medium, provided the original work is properly cited.

\begin{abstract}
The main objective of this paper is to describe and interpret an SIR (Susceptible-Infectious-Recovered) epidemic model though a logistic equation, which is parameterized by a Malthusian parameter and a carrying capacity parameter, both being time-varying, in general, and then to apply the model to the COVID-19 pandemic by using some recorded data. In particular, the Malthusian parameter is related to the growth rate of the infection solution while the carrying capacity is related to its maximum reachable value. The quotient of the absolute value of the Malthusian parameter and the carrying capacity fixes the transmission rate of the disease in the simplest version of the epidemic model. Therefore, the logistic version of the epidemics' description is attractive since it offers an easy interpretation of the data evolution especially when the pandemic outbreaks. The SIR model includes recruitment, demography, and mortality parameters, and the total population minus the recovered population is not constant though time. This makes the current logistic equation to be time-varying. An estimation algorithm, which estimates the transmission rate through time from the discrete-time estimation of the parameters of the logistic equation, is proposed. The data are picked up at a set of samples which are either selected by the adaptive sampling law or allocated at constant intervals between consecutive samples. Numerical simulated examples are also discussed.
\end{abstract}

\section{Introduction}

Epidemic mathematical models under different formal frameworks are of major interest along the last years $[1,2]$. Some of such models rely on the dynamic aspects of the disease evolution and are stated in terms of differential, difference, and differential/difference hybrid equations, dynamic systems, and/or control theory (so as to deal with vaccination and treatment intervention strategies) [3-18], computation tools [7], information theory, [19-22], etc. On the contrary, some of the above invoked modelling techniques combine several analysis and design tools. See, for instance, $[19,20]$ and some references therein are related to designs, the optimality of the controls, and the use of fractional- order models. There are also epidemic models available which consider the spatial-temporal spread of the disease. For instance, several neighboring domains with diversity of populations are considered in [23] concerning the HIV/AIDS spread. It is considered that different levels of awareness are present in different groups which might affect, for instance, to the hardness of the actions to be taken for transmission prevention. In [24], a Latin hypercube sampling method is discussed to calculate relevant reproduction numbers from distributions of some relevant model-related parameters.

It can be pointed out that continuous-time models are widely used since they are more tractable mathematically than the discrete models while having a direct physical interpretation [25]. In particular, the positivity of the solution under nonnegative initial conditions can be proved 
from the differential system describing the model without the use of extradiscretization adjusting parameters. Furthermore, it is a direct task to pick up values from the solution at appropriate sampling points (for instance, daily or weekly) of the continuous-time solution of the model, provided that such an information is needed for storing statistic data or in order to decide the implementation of some vaccination or other alternative public intervention controls such as partial quarantines or confinements. On the contrary, the use of discrete models has been shown to be specifically useful in the context of dissemination of diseaserelated information such as its current state or evolution tendency, for instance, through social networks. That associated information can help to prevent or to reduce possible foreseen outbreaks [26].

A basic goal is to investigate and predict the evolution of infectious diseases as well as to focus on how the public interventions, for example, quarantines or vaccination and treatment controls can mitigate their outbreaks and uncontrolled propagations. See, for instance, $[1,2]$ and $[11,13,15,17,18,27,28]$. A beneficial effect of quarantine interventions on a part of a targeted subpopulation, in particular, either fractions or the susceptible subpopulation or fractions of the infectious one is their "removal" from its associate compartment. In this way, the most apparent effect in the disease evolution through time is a reinitialization of the corresponding trajectory solution under fewer numbers of contagious contacts. A second beneficial effect is that the disease transmission rate decreases to more moderate levels as a result of the reduction in the contagion contacts. Those concerns have been seen to be relevant concerning the evolution of the current COVID-19 pandemic. See, for instance, [29-46] and some of the references therein. In particular, an SEIR model is proposed in [41] for the COVID-19 pandemic. Such a model includes delayed resusceptibility caused by the infection. Also, a kind of autoregressive model average model (ARMA), so-called an ARIMA model, for prediction of COVID-19 is presented and simulated in [42] for the data of several countries. The effects of different phases of quarantine actions in the values of the transmission rate are studied in [43], see also [46], for a comparative and exhaustive discussion of related simulated numerical results on the COVID-19 outbreak in Italy.

The related existing bibliography is abundant and very rich including a variety of epidemic models with several coupled subpopulations included as an elementary starting basis of the susceptible, the infectious, and the recovered ones in the simpler SIR epidemic models. Further generalizations lead to the so-called SEIR models which include the exposed subpopulation, that is, those who do not have external symptoms yet, as a new subpopulation. More complex models, such as SEIADR-type models, which include the asymptomatic infectious and the infective dead subpopulations, have been designed and analyzed, for instance, Ebola disease $[15,16]$.

On the contrary, it is well known that the celebrated logistic equation of Velhulst is a model of population growth which is parameterized by two constant parameters, namely, the Malthusian parameter and the carrying capacity, see, for instance, [47]. The main objective of this paper is to propose and to link a SIR (susceptible-infectious-recovered) with recruitment and demography to a proposed time-varying logistic equation parameterized by a time-varying carrying capacity and a time-varying Malthusian parameter and whose solution is attractive and of simple interpretation. The methodological analysis key point is to compare the current SIR epidemic model with eventual recruitment, demography, and disease-related mortality parameters to the socalled nominal (or reference) case, where those parameters are zeroed and, in addition, the recovered are considered constant being equal to their initial values along with the time interval of interest. The total reference population is also constant due to the absence of recruitment, demography, and disease-related mortality. It turns out that the above nominal logistic-type model can work efficiently along certain transient periods of time (of the order of weeks) when the infection is growing up very fast, but there are no still recovered subpopulation, and the total population does not vary significantly. The nominal case might be described, equivalently, by a time-invariant logistic equation which has a unique solution for the infectious depending on the initial conditions and on two constant (reference Malthusian and reference carrying capacity) parameters which depend on the transmission rate, the recovery rate, and the initial levels of total population and immune subpopulation. In particular, the Malthusian parameter quantifies the rate of either growth or decrease of the infection and the (infection) carrying capacity fixes the maximum level of such an infection along its growing time period (say, the maximum of the infectious subpopulation).

Then, a most complex current SIR model is described through the abovementioned time-varying logistic equation whose solution is characterized as that of the nominal one plus an error function which depends on the new added parameters (recruitment rate, demography rate, and diseaserelated mortality). A practical way of monitoring the timevarying logistic equation in a simple way is to reinitialize it by simple resetting of initial conditions, when necessary, and to keep its parameterization in operation along time intervals of appropriate moderate adaptive lengths before the next resetting. For each updated parameterization, the logistic equation is run as time-invariant until its next resetting. In this way, the current Malthusian parameter and carrying capacity are modelled by piecewise constant functions whose values are updated, in general, at a sequence of sampling instants subject, in general, to nonuniform intersample periods. Such periods are adapted to the infection variation so that the larger the infection variation, the smaller the intersample period.

The paper is organized as follows. Section 2 states the SIR (susceptible subpopulation S-infectious subpopulation I-recovered subpopulation R) epidemic model with population recruitment, demography, and disease mortality and establishes and proves its positivity and boundedness properties. Section 3 is split into several sections. The first one rewrites the model under the equivalent form of a timevarying logistic equation parameterized by a the Malthusian parameter, which defines the exponential order of the 
solution form, and a carrying capacity which is related to the maximum attainable infection level along its growing period. Both parameters are, in general, time-varying functions which are defined based on the primary model parameters and the values of the subpopulations through time. Section 3.2 discusses the description of a simplified version of the above epidemic model to the light of a time-invariant logistic equation parameterized by constant values of the Malthusian parameter and the carrying capacity. It also assumes that the increment of the total population over the recovered subpopulation remains constant. Such a time-invariant logistic equation is referred to as the nominal (or reference) logistic equation. The simplified version of the SIR model, rewritten equivalently as the nominal logistic equation, is recruitment, demography, and mortality free. Those simplifications are reasonable along periods lasting some months since the disease-associated mortality is usually small compared to the total population, while the recruitment and demography rates are also usually small related to the total population amounts along short periods of time compared with the species life expectation. An important motivating result of the problem approach is that the carrying capacity is the quotient between the absolute Malthusian parameter and the carrying capacity. In this way, the nominal logistic equation characterizes a constant disease transmission rate for the simplified SIR model of no public interventions of confinement or quarantine type is performed. In the same way, the current time-varying logistic equation establishes a time-varying disease transmission rate for the more complex model which also depends on such public interventions, in the case where they are performed. Section 3.3 obtains in a closed form the solution of the time-varying logistic equation related to the nominal one and computes also the worst-case of the error through time among both of them. It also discusses a technique to evaluate the current solution along with a set of samples which are updated through adaptive sampling laws which decrease the intersampling period as the infection variation between consecutive samples increases and vice versa. Finally, Section 3.4 gives a pseudocode algorithm to implement the above ideas to estimate the Malthusian and carrying capacity time-varying parameters from registered infection data on sequences of time instants. With those parameters, the timecarrying capacity is also sample-dependent calculated and its averaged value along the time period of each public intervention (quarantines, confinements, de-escalating postquarantine phases, or public intervention void) is also estimated. Section 4 is devoted to perform some numerical examples as well as to discuss the obtained results with special emphasis on the influences on the infection propagation of partial or total quarantines of some or all the subpopulations or de-escalating phases after quarantines. Model parameterizations with available recorded data related to the COVID-19 pandemic are used for the testing process and the related transmission rate obtained. Finally, Section 5 ends the paper. Some auxiliary calculations related to the estimations of the Malthusian parameter and the carrying capacity concerned with a relevant step of the estimation algorithm of Section 34 are given in Appendix A.

\section{The SIR Epidemic Model and Its Positivity and Boundedness Properties}

Consider the following SIR (susceptible $S$-infectious I-recovered $R$ ) epidemic model with demography:

$$
\begin{gathered}
\dot{S}(t)=v-(\mu+\beta I(t)) S(t), \quad S(0)=S_{0}, \\
\dot{I}(t)=(\beta S(t)-\gamma-\mu) I(t), \quad I(0)=I_{0}, \\
\dot{R}(t)=(1-\rho) \gamma I(t)-\mu R(t), \quad R(0)=R_{0},
\end{gathered}
$$

subject to arbitrary nonnegative finite initial conditions, i.e., $\min \left(S_{0}, I_{0}, R_{0}\right) \geq 0$, where $\beta$ is the disease transmission rate $\mu$ and $\rho \in[0,1]$ are, respectively, the natural and disease-related mortality rates, and $\nu(\geq \mu)$ is the population recruitment rate. The total population is $N(t)=S(t)+I(t)+R(t)$ whose derivative with respect to time is obtained by summing-up (1)-(3):

$$
\begin{aligned}
& \dot{N}(t)=\nu-\mu N(t)-\rho \gamma I(t), \\
& N(0)=N_{0}=S_{0}+I_{0}+R_{0} .
\end{aligned}
$$

Denote in the following $\mathbf{R}_{+}=\{z \in \mathbf{R}: z>0\}$, $\mathbf{R}_{0+}=\{z \in \mathbf{R}: z \geq 0\}=\mathbf{R}_{+} \cup\{0\}$. The subsequent result relies on the positivity and boundedness of all the solution of (1)-(3) under any given finite nonnegative initial conditions.

Theorem 1. Model (1)-(3) is positive, in the sense that $\forall t \in \mathbf{R}_{0+}$ and globally stable, that is, the solutions of (1)-(3) are bounded for all time for any given finite nonnegative initial conditions. As a result, it holds that $\lim \sup _{t \rightarrow \infty}\left(\sup _{0 \leq \xi \leq t} I(\xi)\right) \leq \nu / \gamma$.

Proof. The solution of (2) is $I(t)=e^{\beta \int_{0}^{t} S(\xi) \mathrm{d} \xi-(\gamma+\mu) t} I_{0} \geq 0$; $\forall t \in \mathbf{R}_{0+}$, since $I_{0} \geq 0$. Therefore, $I: \mathbf{R}_{0+} \longrightarrow \mathbf{R}_{0+}$, for any given nonnegative initial conditions. On the contrary, since $S_{0} \geq 0$ and $S: \mathbf{R}_{0+} \longrightarrow \mathbf{R}_{0+}$ are everywhere continuous (since it is everywhere time-differentiable), it is nonnegative until $S\left(t_{1}\right)=0$, for some $t_{1} \geq 0$, in the event that such a $t_{1}$ exists. However, by inspecting (1), one gets $\dot{S}\left(t_{1}\right)=v \geq 0$ (even if the model is recruitment-free, i.e., if $v=0)$. Then, $S\left(t_{1}^{+}\right) \geq 0$. Therefore, no $t \in \mathbf{R}_{0+}$ can exist such that $S(t)<0$. Therefore, $S: \mathbf{R}_{0+} \longrightarrow \mathbf{R}_{0+}$ for any given nonnegative initial conditions. On the contrary, the solution of (3) is nonnegative in any interval $\left[0, t_{2}\right)$ under any nonnegative initial conditions such that $R\left(t_{2}\right)=0$, but then $\dot{R}\left(t_{2}\right)=(1-\rho) \gamma I\left(t_{2}\right) \geq 0$ so that no $t \in \mathbf{R}_{0+}$ exists such that $R(t)<0$ and $R$ : $\mathbf{R}_{0+} \longrightarrow \mathbf{R}_{0+}$. It has been proved that the solutions of (1)-(3) are always nonnegative for any given nonnegative initial conditions. The total population has, from (4), the following solution equation:

$$
\begin{aligned}
N(t) & =S(t)+I(t)+R(t) \\
& =e^{-\mu t} R_{0}+\int_{0}^{t} e^{-\mu(t-\xi)}(\nu-\gamma I(\xi)) \mathrm{d} \xi \geq 0, \quad \forall t \in \mathbf{R}_{0+},
\end{aligned}
$$

so that one has, for any $t_{0}, t\left(\geq t_{0}\right) \in \mathbf{R}_{0+}$, that 


$$
\begin{aligned}
\int_{t_{0}}^{t} e^{-\mu(t-\xi)} I(\xi) \mathrm{d} \xi & \leq \int_{0}^{t} e^{-\mu(t-\xi)} I(\xi) \mathrm{d} \xi \\
& \leq \frac{1}{\gamma}\left[e^{-\mu t} R_{0}+\frac{\nu}{\mu}\left(1-e^{-\mu t}\right)\right]
\end{aligned}
$$

so that, for any given finite $\theta \in \mathbf{R}_{+}$, one has by using the mean value theorem that, for any strictly increasing sequence $\left\{t_{n}\right\}_{n=0}^{\infty} \subset \mathbf{R}_{0+}$ and some sequence $\left\{\varsigma_{n}\right\}_{n=0}^{\infty} \subset\left(t_{n+1}, t_{n}\right)$,

$$
\int_{t_{n}}^{t_{n}+\theta} e^{-\mu\left(t_{n}-\xi\right)} I(\xi) \mathrm{d} \xi=\frac{\theta\left(1-e^{-\mu \theta_{n}}\right)}{\mu} I\left(\varsigma_{n}\right) \leq \frac{\nu}{\gamma \mu},
$$

so that

$$
\limsup _{n \rightarrow \infty} I\left(\varsigma_{n}\right) \leq \frac{\nu}{\gamma \theta}, \quad \forall t \in \mathbf{R}_{0+} .
$$

Since $I: \mathbf{R}_{0+} \longrightarrow \mathbf{R}_{0+}$ is continuous and $\theta$ finite, one concludes that lim $\sup _{n \rightarrow \infty} I\left(t_{n}\right) \leq \nu / \gamma \theta$, and under the same reasoning, one gets $\limsup _{t \rightarrow \infty} I(t) \leq \nu / \gamma \theta$. Since $I: \mathbf{R}_{0+} \longrightarrow \mathbf{R}_{0+}$ is continuous, it cannot be unbounded on any finite time interval $\left[0, t_{1}\right)$ so that the above ultimate boundedness property as time tends to infinity also implies the boundedness of $I: \mathbf{R}_{0+} \longrightarrow \mathbf{R}_{0+}$ in $[0, \infty]$.

For any nonnegative initial conditions and then $I: \mathbf{R}_{0+} \longrightarrow \mathbf{R}_{0+}$ is bounded. This implies from (5) that

$$
N(t) \leq N_{0}+\frac{1}{\mu}\left(\nu+\gamma \sup _{t \geq 0} I(t)\right)<+\infty .
$$

On the contrary, one has from (2) that the (empty or nonempty, connected, or nonconnected) real interval

$$
U_{S}=\left\{t \in \mathbf{R}_{0+}: S(t)>\frac{\gamma+\mu}{\beta}\right\}=\bigcup_{i \in \mathbf{Z}_{0+}}\left(t_{i}, t_{i+1}\right)
$$

has a Lebesgue measure $0 \leq \operatorname{Lb}\left(U_{S}\right)<+\infty$ (zero if it is empty) and finite since otherwise, $\lim _{t \rightarrow \infty} I(t)=+\infty$ which contradicts the boundedness of $I: \mathbf{R}_{0+} \longrightarrow \mathbf{R}_{0+}$ according to (8). Then, the complementary set $U_{S}$ in $\mathbf{R}_{0+}$ is

$$
\begin{aligned}
\mathbf{R}_{0+} \backslash U_{S} & =\left\{t \in \mathbf{R}_{0+}: S(t) \leq \frac{\gamma+\mu}{\beta}\right\} \\
& \left.=\overline{\bigcup_{i \in \mathbf{Z}_{0+}}\left(t_{i}, t_{i+1}\right)}=\bigcap_{i \in \mathbf{Z}_{0+}}\right] t_{i}, t_{i+1}[,
\end{aligned}
$$

which has $0 \leq L b\left(\mathbf{R}_{0+} \backslash U_{S}\right)=+\infty$ and, therefore, $S: \mathbf{R}_{0+} \longrightarrow \mathbf{R}_{0+} \backslash U_{S}$ is bounded while $S: \mathbf{R}_{0+} \longrightarrow U_{S}$ cannot be unbounded since $S: \mathbf{R}_{0+} \longrightarrow \mathbf{R}_{0+}$ is continuous and $U_{S}$ has a finite Lebesgue measure. As a result, $S: \mathbf{R}_{0+} \longrightarrow \mathbf{R}_{0+}$ is bounded for any nonnegative initial conditions. Since $S, I, R, N: \mathbf{R}_{0+} \longrightarrow \mathbf{R}_{0+}$ and $S, I, N: \mathbf{R}_{0+} \longrightarrow \mathbf{R}_{0+}$ are bounded, then

$$
0 \leq R(t)=N(t)-S(t)-I(t)<+\infty, \quad \forall t \in \mathbf{R}_{0+},
$$

and $R: \mathbf{R}_{0+} \longrightarrow \mathbf{R}_{0+}$ is bounded. The theorem has been fully proved.

\section{The Epidemic Model in a Logistic Equation Form}

3.1. "Ad hoc" Time-Varying Logistic Equation. Verhulst's or logistic equation is a celebrated model of population growth which was proposed in 1838 by Pierre Verhulst (1804-1849). It can also be considered in an "ad-hoc" time-varying version to describe the epidemic model of the above section under a certain modification of the basic logistic equation after replacing $S(t)=N(t)-I(t)-R(t)$ in (2). Thus, one gets the following infectious solution with the contribution to its dynamics of the susceptible and recovered subpopulations:

$$
\begin{aligned}
\dot{I}(t) & =\beta(N(t)-I(t)-R(t)) I(t)-(\gamma+\mu) I(t) \\
& =-\beta I^{2}(t)+[\beta(N(t)-R(t))-(\gamma+\mu)] I(t) \\
& =-\frac{I^{2}(t)}{a(t) c(t)}+\frac{I(t)}{a(t)}, \quad \forall t \in \mathbf{R}_{0+},
\end{aligned}
$$

where $a(t) c(t)=1 / \beta$, and

$$
\begin{aligned}
& a(t)=\frac{1}{\beta(N(t)-R(t))-\gamma-\mu}, \\
& c(t)=\frac{1}{\beta|a(t)|}=\left|N(t)-R(t)-\frac{\gamma+\mu}{\beta}\right|, \quad \forall t \in \mathbf{R}_{0+} .
\end{aligned}
$$

The subsequent result will be then used related to the interpretation of the epidemic models (1)-(3) as a timevarying logistic equation related to a nominal time-invariant one, in the case that $S(t)+I(t) \geq S_{0}+I_{0}$ for all $t \in \mathbf{R}_{0+}$.

Proposition 1. The following results hold for a normalized model (1)-(3) at the initial time, that is, if $N_{0}=1$

(i) The inequality

$$
\begin{aligned}
& S(t)+I(t)=N(t)-R(t) \geq S_{0}+I_{0}=N_{0}-R_{0} \\
& \text { is guaranteed for a given } t \in \mathbf{R}_{+} \text {if } \\
& \int_{0}^{t} e^{-\mu(t-\xi)}(\nu-\gamma I(\xi)) \mathrm{d} \xi \geq\left(1-e^{-\mu t}\right)\left(N_{0}-R_{0}\right) .
\end{aligned}
$$

and the inequality is reversed, namely, for a given $t \in \mathbf{R}_{+}$,

$S(t)+I(t)=N(t)-R(t) \leq S_{0}+I_{0}=N_{0}-R_{0}$

if

$\int_{0}^{t} e^{-\mu(t-\xi)}(\nu-\gamma I(\xi)) \mathrm{d} \xi \leq\left(1-e^{-\mu t}\right)\left(N_{0}-R_{0}\right)$. 
(ii) The above inequality is guaranteed in $[0, t]$ for any given $t \in \mathbf{R}_{+}$if

$$
\sup _{0 \leq \xi \leq t} I(\xi) \leq \frac{\nu-\mu}{\gamma}
$$

so that it is guaranteed in $[0, \infty)$ as well. The above inequality also guarantees that $\liminf _{t \rightarrow \infty}(S(t)+I(t)) \geq S_{0}+I_{0}$ is fulfilled since

$$
\limsup _{t \rightarrow \infty}\left(\sup _{0 \leq \xi \leq t} I(\xi)\right) \leq \frac{\nu-\mu}{\gamma} .
$$

Proof. The solution to (3) is

$$
R(t)=e^{-\mu t} R_{0}+\gamma(1-\rho) \int_{0}^{t} e^{-\mu(t-\xi)} I(\xi) \mathrm{d} \xi, \quad \forall t \in \mathbf{R}_{0+},
$$

which together with (5) yields

$$
N(t)-R(t)=e^{-\mu t}\left(N_{0}-R_{0}\right)+\int_{0}^{t} e^{-\mu(t-\xi)}(\nu-\gamma I(\xi)) \mathrm{d} \xi, \quad \forall t \in \mathbf{R}_{0+} .
$$

Note that $S(t)+I(t)=N(t)-R(t) \geq S_{0}+I_{0}=N_{0}-R_{0}$ for some $t \in \mathbf{R}_{0+}$ if and only if

$$
\int_{0}^{t} e^{-\mu(t-\xi)}(\nu-\gamma I(\xi)) \mathrm{d} \xi \geq\left(1-e^{-\mu t}\right)\left(N_{0}-R_{0}\right),
$$

and the first part of Property (i) is proved. The reversed inequality and associated condition are proved "mutatismutandis." On the contrary, the above inequality holds on $[0, t]$ if

$$
\frac{\gamma\left(1-e^{-\mu t}\right)}{\mu} \sup _{0 \leq \xi \leq t} I(\xi) \leq \frac{\nu\left(1-e^{-\mu t}\right)}{\mu}-\left(1-e^{-\mu t}\right)\left(S_{0}+I_{0}\right),
$$

for a normalized model at the initial conditions, that is, for $S_{0}+I_{0} \leq N_{0}=1 \quad$ if $\sup _{0 \leq \xi \leq t}(\xi) \leq \nu-\mu / \gamma$. Also, $\liminf _{t \rightarrow \infty}(S(t)+I(t)) \geq S_{0}+I_{0}$ $\lim \sup _{t \rightarrow \infty} \int_{0}^{t} e^{-\mu(t-\xi)} I(\xi) \mathrm{d} \xi \leq 1 / \gamma\left(\nu / \mu-t S_{0} n-q I_{0}\right)$

which is guaranteed if $\limsup \sup _{t \rightarrow \infty} \int_{0}^{t} e^{-\mu(t-\xi)} I(\xi) \mathrm{d} \xi \leq \nu-\mu / \gamma \mu$ which is guaranteed if $\lim \sup _{t \rightarrow \infty}\left(\sup _{0 \leq \xi \leq t} I(\xi)\right) \leq \nu-\mu / \gamma$. Property (ii) has been proved.

From the nonnecessary normalized model at the initial conditions, one has the following result which follows directly from (24) in the proof of Proposition 1:

Proposition 2. $S(t)+I(t) \geq S_{0}+I_{0}$ holds for all $t \in\left[0, t_{a}\right]$, provided that $S_{0}+I_{0} \leq \nu / \mu$ if

$$
\sup _{0 \leq t \leq t_{a}} I(t) \leq \frac{1}{\gamma}\left(\nu-\mu\left(S_{0}+I_{0}\right)\right) \text {. }
$$

3.2. Nominal Logistic Equation. Assume that the epidemic models (1)-(3) are considered without recruitment, demography, and mortality, that is, $\nu=\mu=\rho=0$. Thus, one has from (4) and (13) and (14) that $N(t) \equiv 0$ so that $N(t)=N_{0} ; \forall t \in \mathbf{R}_{0+}$. It is also assumed that the total population excess over the recovered subpopulation remains constant. This assumption keeps the logistic equation timeinvariant, for instance, identical to their initial condition excess $N_{0}-R_{0}$. The above hypotheses is reasonable along periods of several months over which the above population/ recovered subpopulation excess does not vary substantially in practice. Thus, the parameterization of the (so-called nominal or reference) logistic equation is time invariant accordingly to

$$
\begin{aligned}
& a(t) \equiv a_{r}=\frac{1}{\beta\left(N_{0}-R_{0}\right)-\gamma}, \\
& c(t) \equiv c_{r}=\frac{1}{\beta\left|a_{r}\right|}=\left|N_{0}-R_{0}-\frac{\gamma}{\beta}\right|,
\end{aligned}
$$

and (13) has the following nominal expression parameterized by (26):

$$
\dot{I}_{r}(t)=\frac{I_{r}(t)}{a_{r}}-\frac{I_{r}^{2}(t)}{a_{r} c_{r}}=\frac{r I_{r}(t)\left(K_{r}-I_{r}(t)\right)}{K_{r}}, \quad I_{r}(0)=I_{0}
$$

where

$$
r=\frac{1}{a_{r}}=\beta\left(N_{0}-R_{0}\right)-\gamma
$$

is the Malthusian parameter, namely, the rate of maximum infection growth which can be positive, negative, or zero, and

$$
K_{r}=r a_{r} c_{r}=c_{r}=\left|N_{0}-R_{0}-\frac{\gamma}{\beta}\right|
$$

is the carrying capacity, namely, the maximum sustainable infectious subpopulation. The normalized by $K_{r}$ differential equation (27) becomes for

$$
\begin{aligned}
\dot{I}_{r n} & =r I_{r n}(t)\left(1-K_{r} I_{n r}(t)\right), \\
I_{r n}(0) & =I_{r n 0}=\frac{I_{r 0}}{K_{r}}
\end{aligned}
$$

where $I_{r n}(t)=I_{r}(t) / K_{r}$ and whose solution is the sigmoid function:

$$
\begin{aligned}
I_{r n}(t) & =\frac{1}{1+\left(I_{r n 0}^{-1}-1\right) e^{-r t}}=\frac{I_{r n o} e^{r t}}{I_{r n o} e^{r t}+\left(1-I_{r n o}\right)} \\
& =\frac{I_{r 0} e^{r t}}{I_{r 0}\left(e^{r t}-1\right)+K_{r}}=\frac{I_{r 0}}{I_{r 0}\left(1-e^{-r t}\right)+K_{r} e^{-r t}} .
\end{aligned}
$$

Then, the unnormalized infectious evolution is 


$$
\begin{aligned}
I_{r}(t)=K_{r} I_{r n}(t) & =\frac{K_{r} I_{r 0}}{I_{r 0}\left(1-e^{-r t}\right)+K_{r} e^{-r t}} \\
& =\frac{c_{r} I_{r 0}}{I_{r 0}\left(1-e^{-t / a_{r}}\right)+c_{r} e^{-t / a_{r}}} \\
& =\frac{c_{r} I_{r 0}}{I_{r 0}+\left(c_{r}-I_{r 0}\right) e^{-t / a_{r}}},
\end{aligned}
$$

and, for $I_{r 0} \neq 0$,

$$
I_{r}(t)=\frac{K_{r}}{1+\left(K_{r} / I_{r 0}-t 1\right) e^{-r t}}=\frac{K_{r}}{1+e^{r\left(t_{M d^{-}} t\right)}}
$$

if $e^{t_{M d^{r}}}=K_{r} / I_{r 0}-1$, namely, if

$$
\begin{aligned}
t_{M d} & =\frac{1}{r} \ln \frac{K_{r}-I_{r 0}}{I_{r 0}}=a_{r} \ln \frac{c_{r}-I_{r 0}}{I_{r 0}} \\
& =\frac{1}{\beta\left(N_{0}-R_{0}\right)-\gamma} \ln \left(\frac{\left|\left(N_{0}-R_{0}\right)-\beta^{-1} \gamma\right|}{I_{r 0}}-1\right) .
\end{aligned}
$$

It also holds that the maximum value of $\dot{I}_{r}(t)$, which is the zero of $\ddot{I}_{r}(t)$ (or the growth inversion point), takes place at $t=t_{M d}$, equation (34), from (33). This is easily seen from the nominal version of the logistic equation (13) with $I(t) \equiv I_{r}(t), a(t) \equiv a_{r}$, and $c(t) \equiv c_{r}$, which leads to

$$
\ddot{I}_{r}(t)=\frac{\dot{I}_{r}(t)}{a_{r}}\left(1-\frac{2 I_{r}(t)}{c_{r}}\right)=0,
$$

which implies that $I_{r}(t)=c_{r} / 2=K_{r} / 2$, if $\dot{I}_{r}(t) \neq 0$, which happens at $t=t_{M d}$ from (33). Therefore, $t_{M d}$ is the growth inversion point time instant. On the contrary, from (27), there is no relative finite maximum value of $I_{r}(t)$ since $\dot{I}_{r}(t)=0$ holds for $t=\infty$ if $r>0$. We have, as basic relations for the key parameters of the nominal logistic equation associated with (1)-(3), the Malthusian parameter (28), the carrying capacity (29), and the growth inversion point (34).

The following result of interest holds.

Proposition 3. The nominal logistic equation has the following properties:

(i) It satisfies the positivity and boundedness conditions of Theorem 1.

(ii) The disease transmission rate satisfies the constraint $\beta=|r| / K_{r}$ so that either $\beta>0$ and $K_{r}$ is finite or $\beta=0$ with $r=-\gamma$ and $K_{r}=\infty$. If $\beta>\gamma / N_{0}-R_{0}$ (respectively, $\beta<\gamma / N_{0}-R_{0}$ ), then $r>0$ (respectively, $r<0$ ). If $\beta=\gamma / N_{0}-R_{0}$, then $r=0$.

(iii) The Malthusian parameter and the carrying capacity increase (respectively, decrease) as the disease transmission rate increases (respectively, decreases). The same property holds with respect to $\beta$ for the growth inversion point time instant if $\beta<1$.

Proof. Property (i) is a corollary of Theorem 1 for the nominal logistic equation which is a particular parameterization of the nominal one. Then, note that the combination of (28) and (29) yields the constraint:

$$
K_{r}=\frac{\left|\beta\left(N_{0}-R_{0}\right)-\gamma\right|}{\beta}=\frac{|r|}{\beta},
$$

which implies that the transmission rate satisfies $\beta=|r| / K_{r}$. The remaining results of Property (ii) follow directly from (28) and (29). On the contrary, note, from (28) and (29) that

$$
\begin{aligned}
r\left(\beta_{2}\right)-r\left(\beta_{1}\right) & =\left(N_{0}-R_{0}\right)\left(\beta_{2}-\beta_{1}\right), \\
K_{r}\left(\beta_{2}\right)-K_{r}\left(\beta_{1}\right) & =\frac{\gamma\left(\beta_{2}-\beta_{1}\right)}{\beta_{1} \beta_{2}},
\end{aligned}
$$

and also one has from (34) that

$$
\begin{aligned}
\frac{\mathrm{d} t_{M d}}{\mathrm{~d} \beta}= & \frac{1}{\beta\left(N_{0}-R_{0}\right)-\gamma} \times \frac{\gamma \beta^{-2} I_{r 0}}{N_{0}-R_{0}-\gamma \beta^{-1}-I_{r 0}} \\
& -\frac{N_{0}-R_{0}}{\left(\beta\left(N_{0}-R_{0}\right)-\gamma\right)^{2}} \times \ln \left(\frac{N_{0}-R_{0}-\gamma \beta^{-1}}{I_{r 0}}-1\right)
\end{aligned}
$$

so that $\mathrm{d} t_{M d} / \mathrm{d} \beta=o\left(\beta^{-3}\right)$. Property (iii) has been proved.

Remark 1. Note from (27) that if $r>0$ (that is if the transmission rate is large enough to satisfy $\left.\beta>\gamma /\left(N_{0}-R_{0}\right)\right)$ and $I_{r 0}<K_{r}$, then $I_{r}(t)$ is strictly increasing until it reaches its maximum value $K_{r}$. If $r$ turns to a negative value, that is if $\beta<\gamma /\left(N_{0}-R_{0}\right)$, then the $I_{r}(t)$ reverses its growth turning to a strictly decreasing profile. The effective way of achieving this behaviour is to decrease the number of effective infectious contacts to the susceptible either by social discipline or eventually via quarantines or confinements.

3.3. Comparison Results of the Logistic Equation with Its Nominal Version. Note that the time-varying logistic equation (13) may be compared to the nominal one (27) by expressing the errors between both current and nominal Malthusian parameters and carrying capacities. For that purpose, define parametrical errors:

$$
\begin{aligned}
& \tilde{a}(t)=a(t)-a_{r}=\frac{1}{\beta(N(t)-R(t))-\gamma-\mu}-\frac{1}{\beta\left(N_{0}-R_{0}\right)-\gamma}, \\
& \tilde{c}(t)=c(t)-c_{r}=\left|N(t)-R(t)-\frac{\gamma+\mu}{\beta}\right|-\left|N_{0}-R_{0}-\frac{\gamma}{\beta}\right|
\end{aligned}
$$

so that

$$
\begin{aligned}
& r(t)=\frac{1}{a(t)}=\frac{1}{a_{r}+\tilde{a}(t)}=\frac{1}{a_{r}}+\widetilde{r}(t)=r+\widetilde{r}(t), \\
& K(t)=c(t)=c_{r}+\tilde{c}(t)=K_{r}+\widetilde{K}(t),
\end{aligned}
$$

where 


$$
\begin{aligned}
& \tilde{r}(t)=r(t)-r_{r}=-\frac{\tilde{a}(t)}{a_{r}+\widetilde{a}(t)}=\frac{\beta\left(N(t)-R(t)-N_{0}+R_{0}\right)-\mu}{\beta(N(t)-R(t))-\gamma-\mu}, \\
& \widetilde{K}(t)=\widetilde{c}(t)=c(t)-c_{r}=\left|N(t)-R(t)-\frac{\gamma+\mu}{\beta}\right|-\left|N_{0}-R_{0}-\frac{\gamma}{\beta}\right|, \\
& \widetilde{b}(t)=\frac{1}{a(t) c(t)}-\frac{1}{a_{r} c_{r}}=-\frac{a_{r} \widetilde{c}(t)+c_{r} \tilde{a}(t)+\widetilde{c}(t) \tilde{a}(t)}{a_{r} c_{r}+\tilde{a}(t) c_{r}+\widetilde{c}(t) a_{r}+\widetilde{c}(t) \tilde{a}(t)} .
\end{aligned}
$$

Thus, (13) can be rewritten as follows:

$$
\begin{aligned}
\dot{I}(t) & =\frac{I(t)}{a(t)}\left(1-\frac{I(t)}{c(t)}\right)=(r+\widetilde{r}(t)) I(t)\left(1-\frac{I(t)}{K_{r}+\widetilde{K}(t)}\right) \\
& =(r+\widetilde{r}(t)) I(t)\left(1-\left(\frac{1}{K_{r}}-\frac{\widetilde{K}(t)}{K_{r}+\widetilde{K}(t)}\right) I(t)\right) \\
& =r I(t)\left(1-\frac{I(t)}{K_{r}}\right)+f(t),
\end{aligned}
$$

where

$$
f(t)=\frac{r \widetilde{K}(t)}{K_{r}+\widetilde{K}(t)} I^{2}(t)+\widetilde{r}(t) I(t)\left(1-\left(\frac{1}{K_{r}}-\frac{\widetilde{K}(t)}{K_{r}+\widetilde{K}(t)}\right) I(t)\right) .
$$

Then, one has that

$$
\begin{aligned}
I(t)= & \left(I_{0}+r \int_{0}^{t} I(\xi)\left(1-\frac{I(\xi)}{K_{r}}\right) \mathrm{d} \xi\right) \\
& +\left(\begin{array}{c}
\int_{0}^{t} \frac{r \widetilde{K}(\xi)}{K_{r}+\widetilde{K}(\xi)} I^{2}(\xi) \mathrm{d} \xi \\
+\int_{0}^{t} \widetilde{r}(\xi) I(\xi)\left(1-\left(\frac{1}{K_{r}}-\frac{\widetilde{K}(\xi)}{K_{r}+\widetilde{K}(\xi)}\right) I(\xi)\right) \mathrm{d} \xi
\end{array}\right) .
\end{aligned}
$$

The first right-hand side term of (45) is the solution of the nominal logistic equation (i.e., (32) or (33)). The second one is the integrated disturbance function $f(\varsigma)$ on $[0, t]$, equation (44), namely, $\int_{0}^{t} f(\xi) \mathrm{d} \xi$ due to the error between the current and nominal solutions. In view of the nominal solution expression (32), one has that (45) can be expressed equivalently as follows:

$$
\begin{aligned}
I(t)= & \frac{c_{r} I_{0}}{I_{0}+\left(c_{r}-I_{0}\right) e^{-t / a_{r}}}+\int_{0}^{t} f(\xi) \mathrm{d} \xi=\frac{c_{r} I_{0}}{I_{0}+\left(c_{r}-I_{0}\right) e^{-t / a_{r}}} \\
& +\left(\int_{0}^{t} \frac{r \widetilde{K}(\xi)}{K_{r}+\widetilde{K}(\xi)} I^{2}(\xi) \mathrm{d} \xi\right. \\
& \left.+\int_{0}^{t} \widetilde{r}(\xi) I(\xi)\left(1-\left(\frac{1}{K_{r}}-\frac{\widetilde{K}(\xi)}{K_{r}+\widetilde{K}(\xi)}\right) I(\xi)\right) \mathrm{d} \xi\right),
\end{aligned}
$$

which can be rewritten for each time instant $t$ from a previous time instant $t-T(t)$ as follows:

$$
\begin{aligned}
I(t)= & \frac{c_{r} I(t-T(t))}{I(t-T(t))+\left(c_{r}-I_{0}\right) e^{-T(t) / a_{r}}} \\
& +\left(\begin{array}{c}
\int_{t-T(t)}^{t} \frac{r \widetilde{K}(\xi)}{K_{r}+\widetilde{K}(\xi)} I^{2}(\xi) \mathrm{d} \xi \\
+\int_{t-T(t)}^{t} \widetilde{r}(\xi) I(\xi)\left(1-\left(\frac{1}{K_{r}}-\frac{\widetilde{K}(\xi)}{K_{r}+\widetilde{K}(\xi)}\right) I(\xi)\right) \mathrm{d} \xi
\end{array}\right) .
\end{aligned}
$$

Assume that, for a given $t_{0} \in \mathbf{R}_{+}$and each $t\left(\geq t_{0}\right) \in \mathbf{R}_{+}$, $T(t)$ is selected so that $\widetilde{K}(t)$ and $\widetilde{r}(t)$ are almost constant with absolute errors less than positive real constants $\varepsilon_{r}$ and $\sup _{t \geq 0}|\widetilde{K}(t)| \leq \varepsilon_{\widetilde{K}}<K_{r}$ (since the current carrying capacity $K(t)=K_{r}+\varepsilon_{\widetilde{K}}$ is positive) in $[t-T(t), t]$. Thus, one has

$$
-\frac{r T(t) \varepsilon_{\widetilde{K}}}{K_{r}-\varepsilon_{\widetilde{K}}} \sup _{t-T(t) \leq \xi \leq t} I^{2}(\xi) \leq \int_{t-T(t)}^{t} \frac{r \widetilde{K}(\xi)}{K_{r}+\widetilde{K}(\xi)} I^{2}(\xi) \mathrm{d} \xi
$$$$
\leq \frac{r T(t) \varepsilon_{\tilde{K}}}{K_{r}} \sup _{t-T(t) \leq \xi \leq t} I^{2}(\xi),
$$

$$
\begin{aligned}
& \mathcal{E}_{\widetilde{r}} \mathcal{E}_{\tilde{K}} T(t) \inf _{t-T(t) \leq \xi \leq t} I(\xi)\left(\frac{K_{r}\left(1+\varepsilon_{\widetilde{K}}\right)+\varepsilon_{\widetilde{K}}}{K_{r}\left(K_{r}-\varepsilon_{\widetilde{K}}\right)} \sup _{t-T(t) \leq \xi \leq t} I(\xi)\right) \\
& \leq \int_{t-T(t)}^{t} \widetilde{r}(\xi) I(\xi)\left(1+\left(\frac{\widetilde{K}(\xi)}{K_{r}+\widetilde{K}(\xi)}-\frac{1}{K_{r}}\right) I(\xi)\right) \mathrm{d} \xi \\
& \leq \varepsilon_{\widetilde{r}} \varepsilon_{\tilde{K}} T(t) \sup _{t-T(t) \leq \xi \leq t} I(\xi)\left(1+\frac{1}{K_{r}} \sup _{t-T(t) \leq \xi \leq t} I(\xi)\right),
\end{aligned}
$$

provided that $\sup _{t \geq 0}|\widetilde{K}(t)| \leq \varepsilon_{\widetilde{K}}<\min \left(K_{r}, 1\right)$. On the contrary, if the incremental carrying capacity related to the nominal one is large enough to satisfy $\inf _{t \geq 0}|\widetilde{K}(t)| \geq K_{r} /\left|K_{r}-1\right|$, which implies also that $\varepsilon_{\widetilde{K}}>1$ while it is also subject to $K_{r}>1$, then

$$
\frac{\widetilde{K}(t)}{K_{r}+\widetilde{K}(t)}-\frac{1}{K_{r}} \leq \frac{\left(K_{r}-1\right) \widetilde{K}(t)-K_{r}}{K_{r}\left(K_{r}+\widetilde{K}(t)\right)} \leq \frac{\varepsilon_{\tilde{K}}\left(K_{r}-1\right)-K_{r}}{K_{r}^{2}}
$$

so that the upper bound in (47) becomes modified as follows:

$$
\varepsilon_{r} \varepsilon \widetilde{K} T(t) \sup _{t-T(t) \leq \xi \leq t} I(\xi)\left(1+\frac{\varepsilon_{\widetilde{K}}\left(K_{r}-1\right)-K_{r}}{K_{r}^{2}} \sup _{t-T(t) \leq \xi \leq t} I(\xi)\right),
$$

Then, one has under the upper bound of (47), for the case when $\sup _{t \geq 0}|\widetilde{K}(t)| \leq \varepsilon_{\widetilde{K}}<K_{r}$ if $\varepsilon=\min \left(\varepsilon_{\tilde{r}}, \varepsilon_{\widetilde{K}}\right)$, that 
(1) Step 0: Choose $t_{0} \geq 0$ and the initial intervention $\mathrm{Phase} \mathrm{Ph}_{0}$ to start to run the procedure.

(2) Smooth the recorded infection curve $I(t)$, if the recorded data are discrete, to make the amended one defined everywhere since the logistic equation used for modelling is continuous through time.

(3) Define $C_{i}$ for $i=1,2,3$ and $T_{m}, T_{M}$ to then run (53)-(55) to generate the sequences IS and ISP of sampling instants and intersampling intervals (or sampling periods) with $T_{M}-T_{m}$ being small enough for the local time-invariance parameterization of the logistic equation to work efficiently.

(4) Step 1: Given the current sampling instant $t_{i} \in \mathrm{IS}$, locate the intervention phase $\mathrm{Ph}_{j}$ to which it belongs. Calculate the intersampling interval $T_{i} \in$ ISP via (54) and some of the equations (55)-(57) and then calculate the next sampling instant $t_{i+1}(\in I S)=t_{i}+T_{i}$

(5) Step 2: Read $I\left(t_{i+1}\right)$ from recorded data

(6) Step 3: The Malthusian parameter and the carrying capacity at time $t_{i}$ are calculated "a posteriori" at time $t_{i+2}=t_{i}+2 T_{i}$ from the registered $I\left(t_{i}\right), I\left(t_{i+1}\right)=I\left(t_{i}+T_{i}\right)$ and $I\left(t_{i}+2 T_{i}\right)$ evaluated at the current and next time instants $t_{i}$ and $t_{i+1}=t_{i}+T_{i}$ and an "a priori" estimation $\widehat{t}_{i+2}=t_{i+1}+T_{i}=t_{i}+2 T_{i}$ of $t_{i+2}$ from equations (A.12)-(A.14) and (A.5) in Appendix A (Remark A.1).

(7) Step 4: Calculate the $\beta\left(t_{i}, t_{i+1}\right)=\left|r\left(t_{i}, t_{i+1}\right) / K\left(t_{i}, t_{i+1}\right)\right|$ (see Proposition 3 (ii)).

(8) Step 5: If $t_{i+1} \in \mathrm{Ph}_{j}$ then make $i \longleftarrow i+1$ and GoTo Step 1. Else GoTo Step 6

(9) Step 6: Calculate the average disease transmission rate of the $\mathrm{Phase}^{\mathrm{Ph}} \beta\left(\mathrm{Ph}_{j}\right)=1 / l_{J} \sum_{t_{i}, t_{i+1} \in \mathrm{Ph}_{j}} \beta\left(t_{i}, t_{i+1}\right)$, make $j \longleftarrow j+1$ and if the whole number of checked phases is unfinished GoTo Step 1. Else GoTo Step 7

(10) Step 7: End

Algorithm 1: Evaluation of the Malthusian parameter and the carrying capacity.

$$
\begin{aligned}
& I(t)=\frac{K_{r} I(t-T(t))}{I(t-T(t))+\left(K_{r}-I(t-T(t))\right) e^{-T(t) r}}+\varepsilon \widetilde{K} T(t) \sup _{t-T(t) \leq \xi \leq t} \\
& I(\xi)\left(\varepsilon_{r}+\frac{K_{r}(r-1)+\varepsilon \widetilde{K}\left(K_{r}-1\right)}{K_{r}^{2}} \sup _{t-T(t) \leq \xi \leq t} I(\xi)\right)+o(\varepsilon T(t)),
\end{aligned}
$$

and one also has, for the case when $|\widetilde{K}(t)| \inf _{t \geq 0} \geq K_{r} /\left|K_{r}-1\right|$, according to (49), that

$$
\begin{aligned}
I(t)= & \frac{K_{r} I(t-T(t))}{I(t-T(t))+\left(K_{r}-I(t-T(t))\right) e^{-T(t) r}} \\
& +\varepsilon_{\bar{K}} T(t) \sup _{t-T(t) \leq \xi \leq t} I(\xi)\left(\varepsilon_{\tilde{r}}+\frac{r+\varepsilon_{r}}{K_{r}} \sup _{t-T(t) \leq \xi \leq t} I(\xi)\right) \\
& +o(\varepsilon T(t)) .
\end{aligned}
$$

On the contrary, note that $\int_{t-T(t)}^{t} \tilde{r}(\xi) I(\xi)\left(1-\left(1 / K_{r}\right.\right.$ $\left.\left.-t \widetilde{K}(\xi) / K_{r}+\widetilde{K}(\xi)\right) I(\xi)\right) \mathrm{d} \xi$ is slowly time varying if $\varepsilon T(t)$ is sufficiently small which is a reasonable approximation for a sufficiently small interval $T(t)$ from the time instant where the initial infection values are picked up until its current calculation. The reason is that this implies in addition that $\varepsilon$ is also sufficiently small which leads to a small variation of $N(\xi)-R(\xi)$ along $[t-T(t), t]$ for a sufficiently small interval $T(t)$ with that additional advantage that the

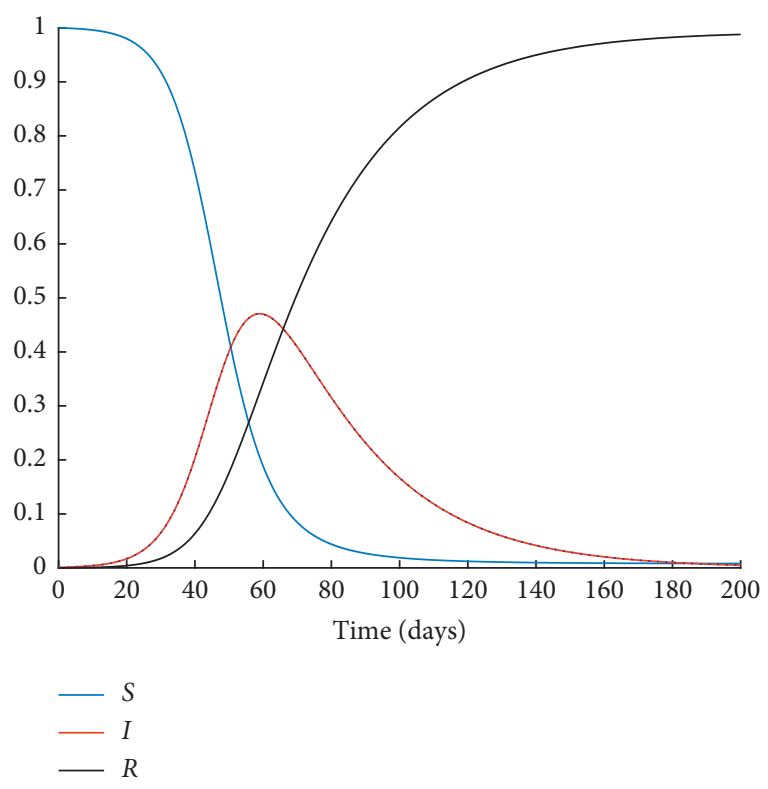

FIGURE 1: Evolution of the SIR model describing the spread of COVID-19 in Italy.

demography-related parameters $\mu$ and $\nu$ are also as small as suited. These considerations suggest that, by choosing the central interval point for such an evaluation of the incremental solution, one gets as an alternative expression to the infectious subpopulation evolution through time that

$$
I(t)=\frac{K(t-n T(t)) I(t-n T(t))}{I(t-n T(t))+(K(t-n T(t))-I(t-n T(t))) e^{-n T(t) r(t-n T(t))}}+\delta(\varepsilon(n T(t)), n T(t)),
$$




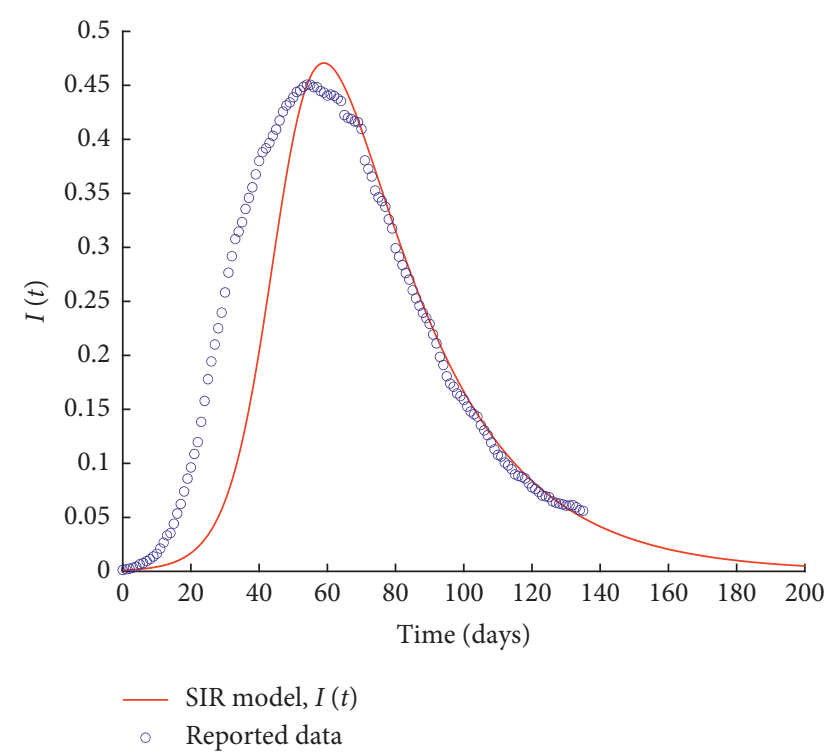

FIGURE 2: Number of active cases per day obtained from the SIR model and from reported data.

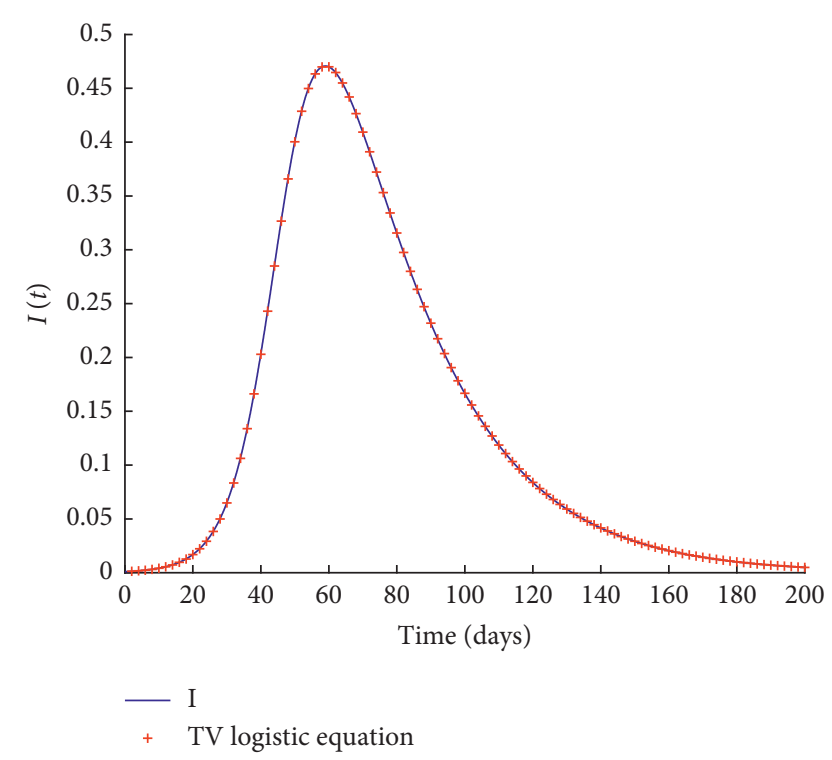

FIGURE 3: Number of active cases per day obtained from the complete SIR model and from the time-varying logistic equation.

for given $n \in \mathbf{Z}_{+}$and $T(t) \in \mathbf{R}_{+}$leading to a performed calculation along a time interval $[t-n T(t), t]$. A further improvement might be performed without difficulty over a sequence, or eventually a finite set IS $\equiv\left\{t_{i}\right\}_{i=0}^{n}$ of sampling instants by using recorded data with a monitored adaptation of the intersampling interval to the rate of variation of the infection curve in such a way that $t_{0}=0, t_{i+1}=t_{i}+T_{i}$, and the intersample period sequence ISP $\equiv\left\{T_{i}\right\}_{i=0}^{n}$ are being updated as follows:

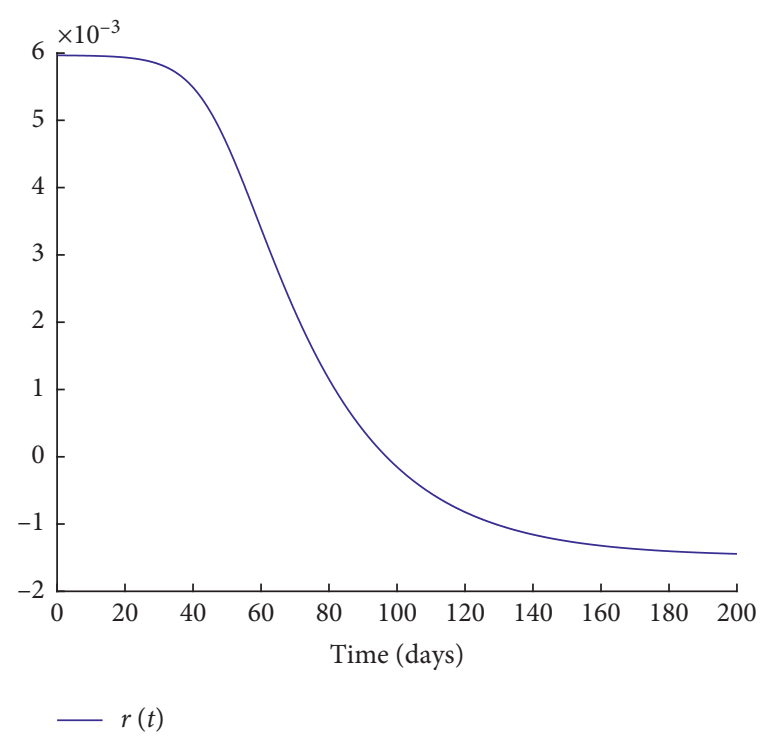

FIGURE 4: Evolution of the Malthusian function, $r(t)$, parameterizing the time-varying logistic equation.

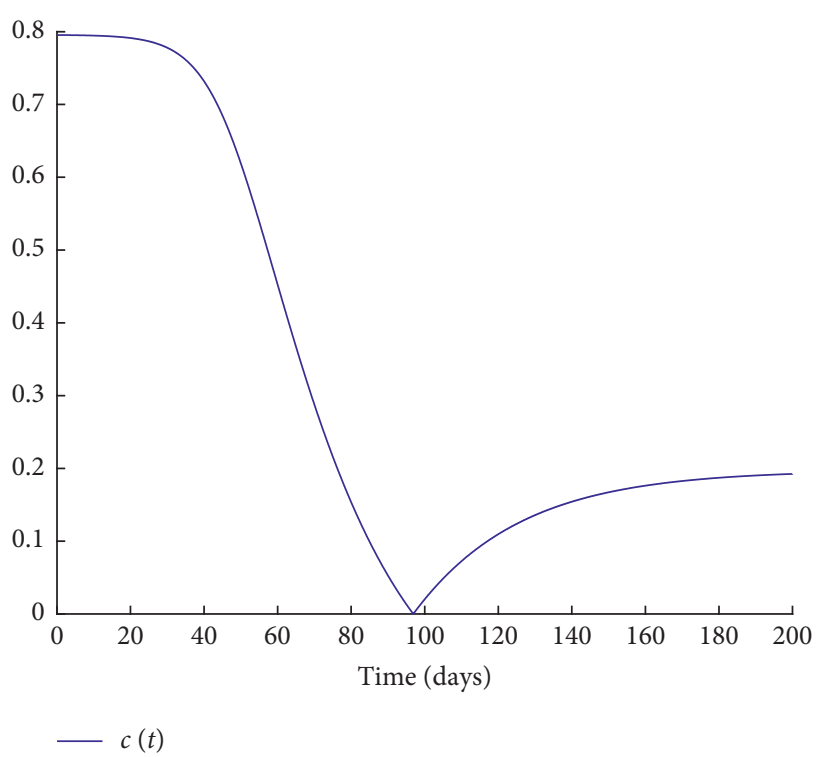

FIGURE 5: Evolution of the carrying capacity, $c(t)$, parameterizing the time-varying logistic equation.

$$
T_{i}= \begin{cases}T_{M}, & \text { if } T_{i a}>T_{M}, \\ T_{i a}, & \text { if } T_{i a} \in\left[T_{m}, T_{M}\right], \\ T_{m}, & \text { if } T_{i a}<T_{m},\end{cases}
$$

where there are a number of possibilities to generate the auxiliary tentative sampling period $T_{i a}$ from the classical adaptive sampling background literature (see [48-51] and related references therein) as, for instance, the three ones which follow: 


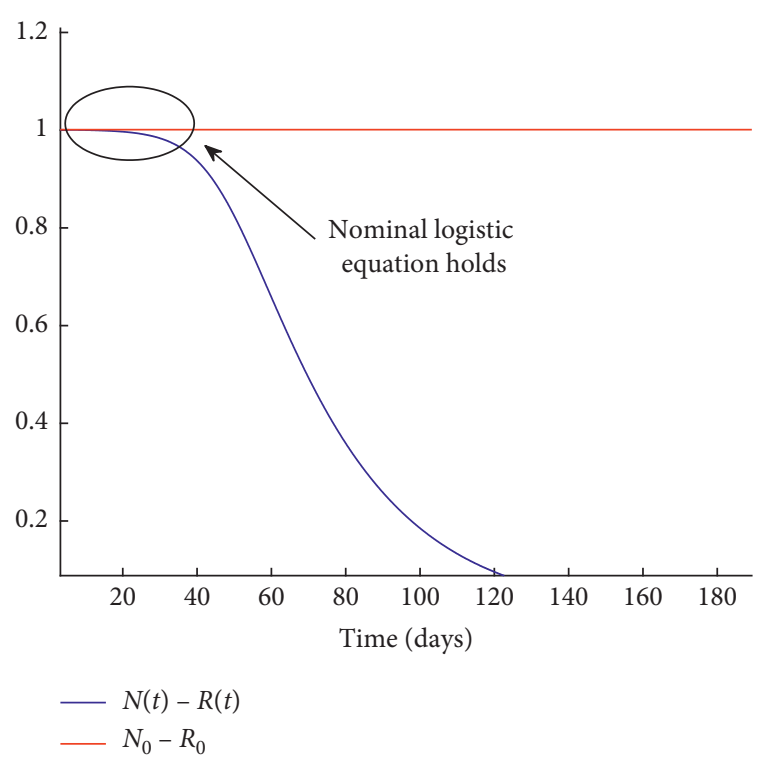

Figure 6: Evolution of $(N(t)-R(t))$ with time.

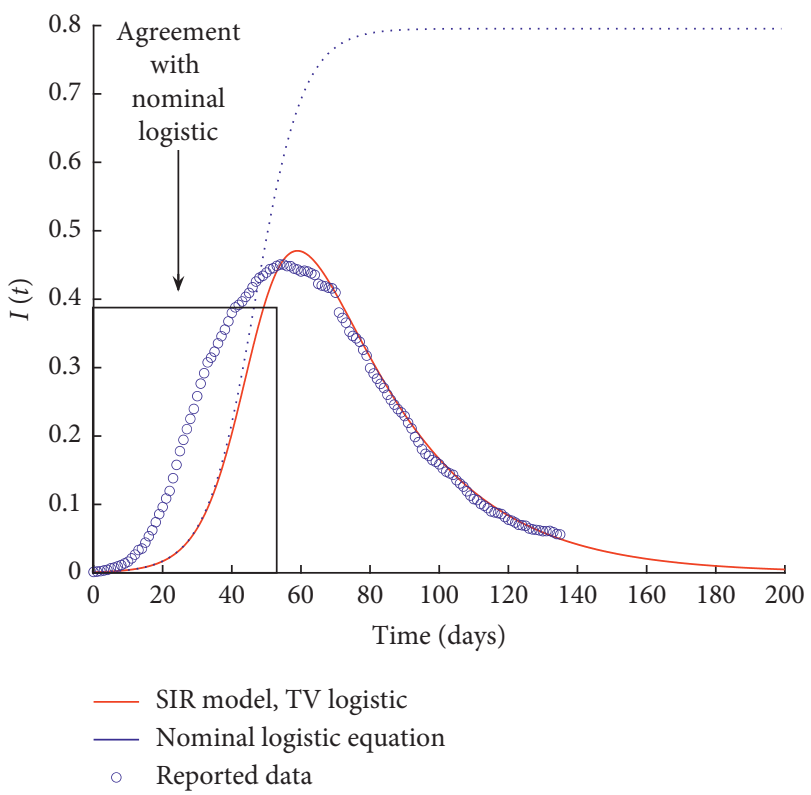

FIgURE 7: Comparison between the SIR model, the time-varying logistic equation, and the nominal one.

$$
\begin{aligned}
T_{i a} & =\frac{C_{1}}{C_{3}+C_{2}\left|\dot{I}\left(t_{i}\right)\right|}, \\
T_{i a} & =\frac{C_{1}}{C_{3}+\left(C_{2}\left|I\left(t_{i}\right)-I\left(t_{i-1}\right)\right| / T_{i-1}\right)}, \\
T_{i a} & =\frac{C_{1}}{C_{3}+C_{2}\left|I\left(t_{i}\right)-I\left(t_{i-1}\right)\right|} .
\end{aligned}
$$

For some prescribed design real constants $C_{1}, C_{2}>0$, $C_{3} \geq 0$, and $T_{m}\left(T_{M}>T_{m}\right)$ with $T_{M}-T_{m}$ being sufficiently small in accordance with the above solution approximation

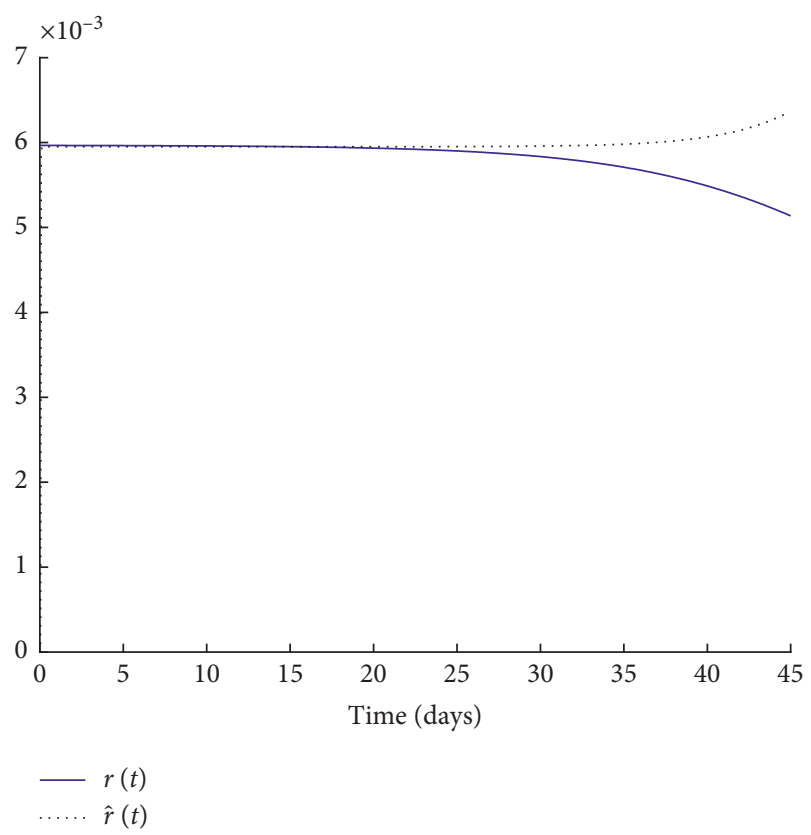

FIgURE 8: Estimation of the Malthusian parameter from the SIR model.

considerations. The adaptive sampling laws (54) and (55) evaluate explicitly the absolute value of time derivative, while the adaptive sampling law (54), which subjects to (56), evaluates it by finite increments. The sampling period is constant (thus, it is nonupdated through time) if $C_{2}=0$, $T_{i a}=T_{m}=C_{1} / C_{3}$. Thus, one gets from (53) the following relation in-between two consecutive sampling instants:

$$
I(t) \cong \frac{K\left(t_{i}\right) I\left(t_{i}\right)}{I\left(t_{i}\right)+\left(K\left(t_{i}\right)-I\left(t_{i}\right)\right) e^{-T_{i} r\left(t_{i}\right)}}, \quad \forall t \in\left[t_{i}, t_{i+1}\right), \forall t_{i} \in \text { IS. }
$$

3.4. Estimation Algorithm of the Malthusian Parameter and the Carrying Capacity along Time. The parameters which characterize the current logistic equation are time varying, but it proposed the following simple algorithm (Algorithm 1) expressed in general pseudocode style. It is assumed that the pandemic is being focused on under different public intervention phases $\left(\mathrm{Ph}_{i}, i \in \mathbf{Z}_{+}\right)$, such as free mobility, confinements, partial quarantines, or different phases of deescalation of quarantines. According to the evaluation of the pandemic growth force, the algorithm identifies from recorded data of the infection curve the Malthusian parameters and the carrying capacity, and then it estimates the averaged disease transmission rate per intervention phase.

Note that the calculation process is repeated for all the pairs of samples $\left(t_{i}, t_{i+1}\right)$ and $\left(t_{i+1}, t_{i+2}\right)$. The transmission rate is calculated for each such a pair from Proposition 3 (ii).

Remark 2. For the pairs $\left(t_{i}, t_{i+1}\right)$ with sampling instants belonging to consecutive phases, we can include the option of considering their associated transmission rates for both 


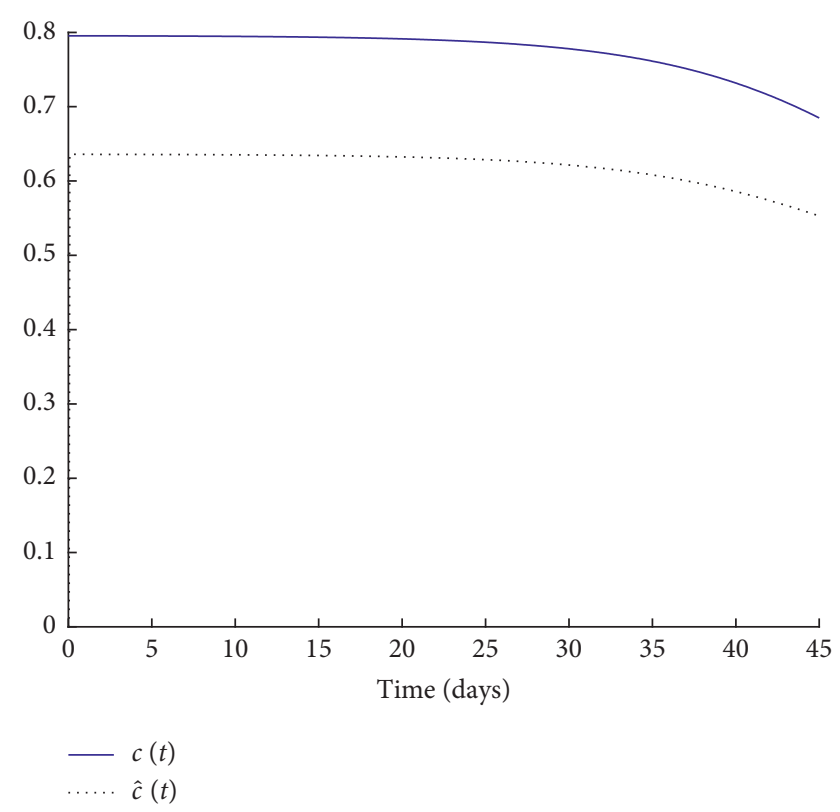

FIGURE 9: Estimation of the carrying capacity from the SIR model.

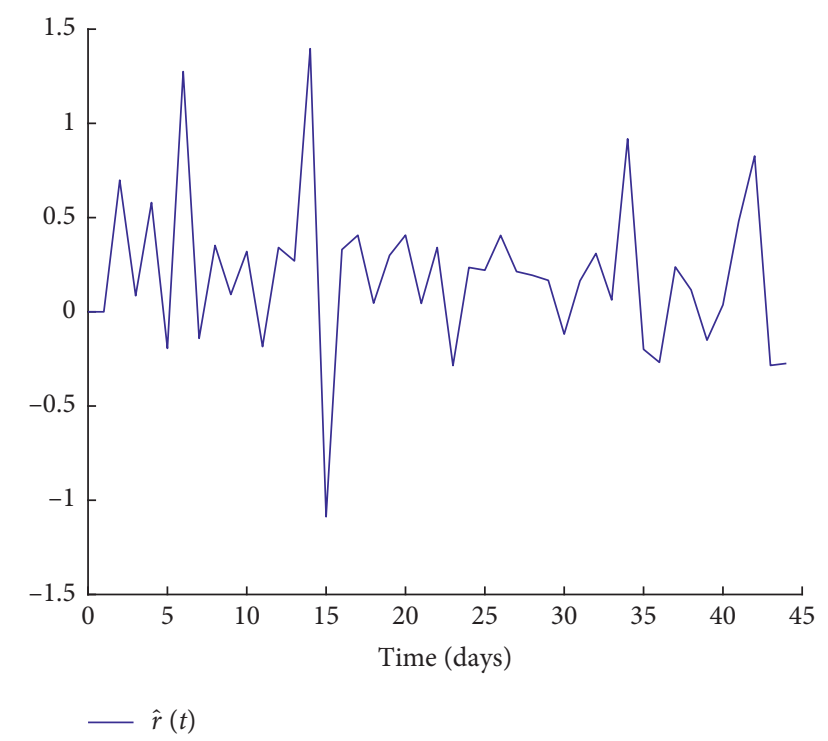

FIGURE 10: Estimation of the Malthusian parameter from reported data (Italy).

phases in Algorithm 1 or for none of them as in the above given version form of Step 6 .

Remark 3. Note that, in Appendix A, two ways of implementing Step 3 of Algorithm 1 to estimate the carrying capacity and the Malthusian parameter are given:

(a) The first one involves the use of infection recorded data after the current evaluation time instant.

(b) The second one is summarized in Remark A.1, and it involves the only use of past data. Concerning the first method, one has to point out that the use of

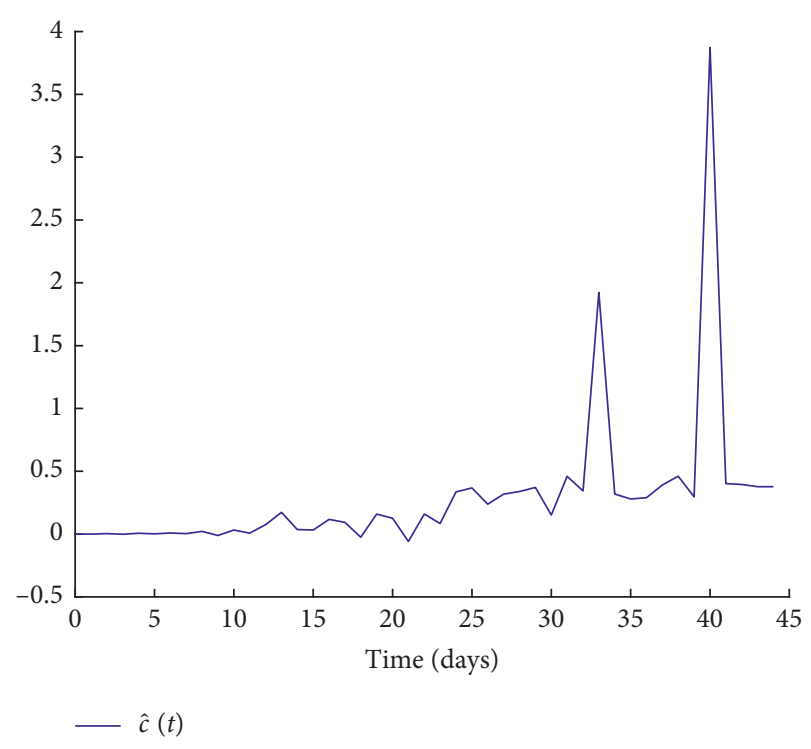

FIGURE 11: Estimation of the carrying capacity from reported data (Italy).

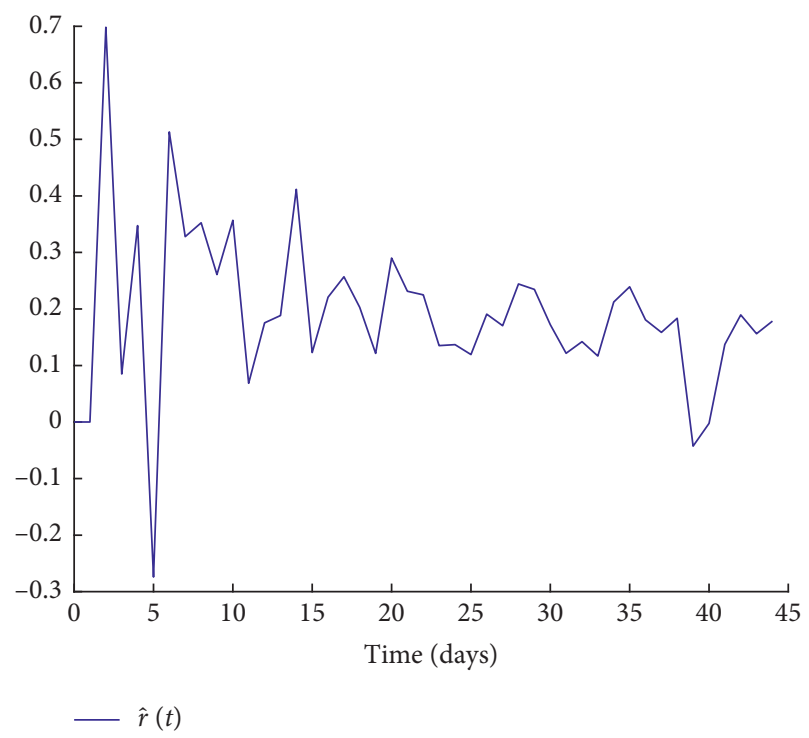

FIgURE 12: Estimation of the Malthusian parameter from filtered reported data (Italy).

predictions can be replaced alternatively by the use for a set of previously recorded data corresponding to the current time instant under checking by the algorithm. Note that both methods can involve the selection of time instants being an integer multiple of the minimum period of supplied data, typically, one day. In this case, the adaptive sampling law just selects a time-varying sampling period which is an integer multiple integer of the mentioned minimum sampling period reported in the recorded data. A particular case is simply to use the minimum sampling period as a constant running sampling period while removing the adaptive sampling law design from Algorithm 1. 


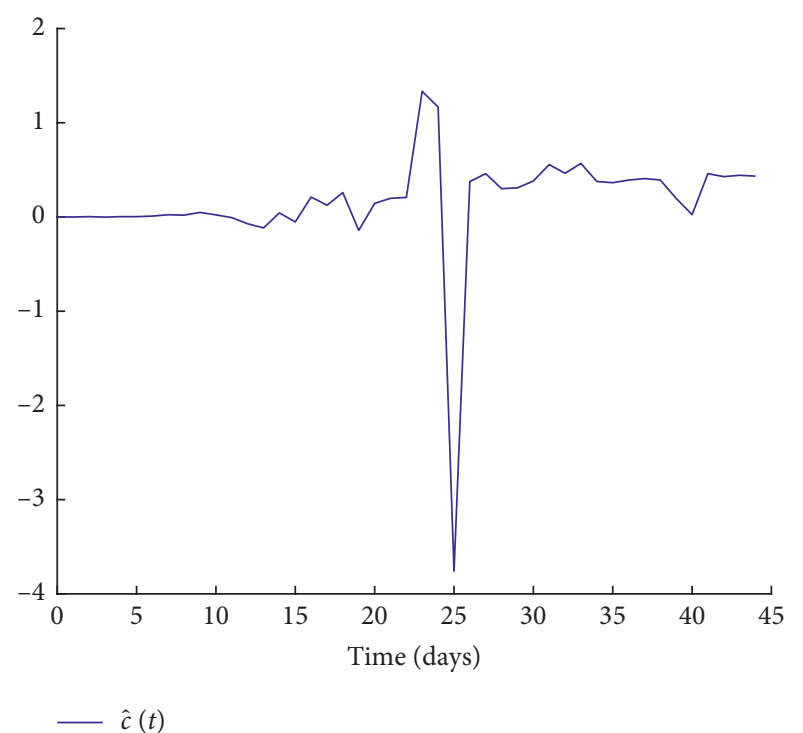

FIGURE 13: Estimation of the carrying capacity from filtered reported data (Italy).

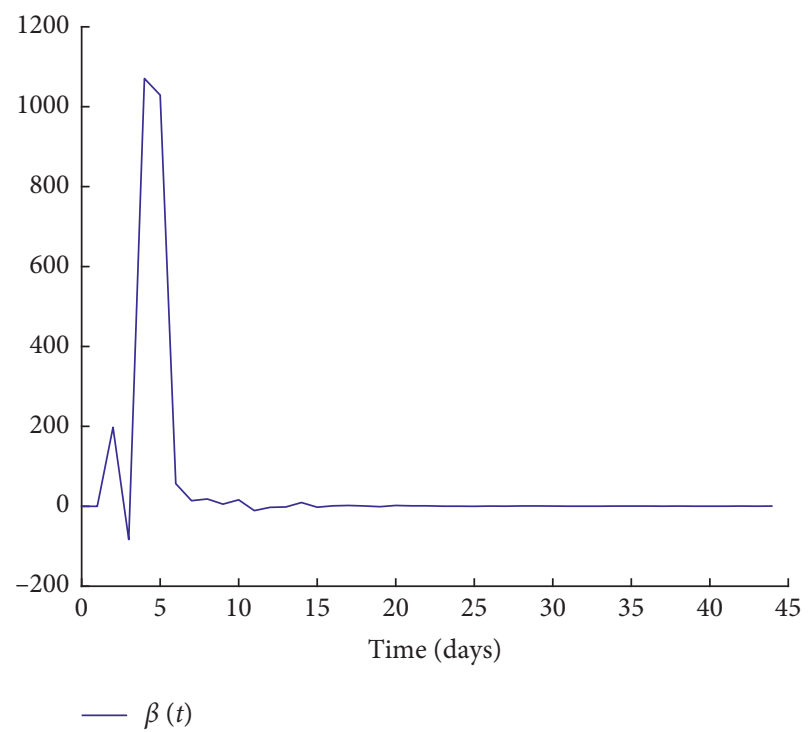

Figure 14: Estimation of $\beta$ from filtered reported data (Italy).

\section{Numerical Examples}

This section is devoted to present some numerical simulation examples illustrating the results discussed in the previous sections. To this end, we consider the case of COVID-19. It is shown in [52] that the spread of COVID-19 in various regions, such as China, South Korea, and Italy (among others) can be appropriately described by a SIR model. For Italy, the SIR model describing the spread of coronavirus is given by $\beta=0.18$, $\gamma=0.037$, and $\rho=\mu=\nu=0$, all of them in units of day ${ }^{-1}$. The initial values for the states are given by $S(0)=240,000, I(0)=$ 240 , and $R(0)=0$. As it is performed in [52], the model is scaled by a factor $f=2.4 \cdot 10^{5}$ in such a way that the scaled initial conditions read $S(0)=1$ and $I(0)=10^{-3}$. The evolution of the scaled SIR model with these parameters is depicted in Figure 1.

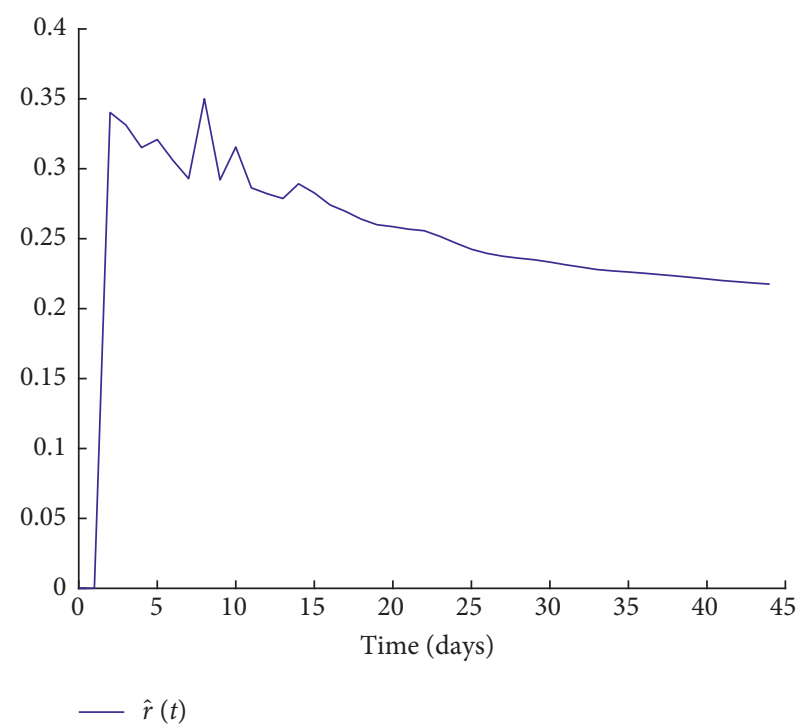

FIGURE 15: Estimation of the Malthusian parameter from reported data (Italy) and lsqcurvefit Matlab function.

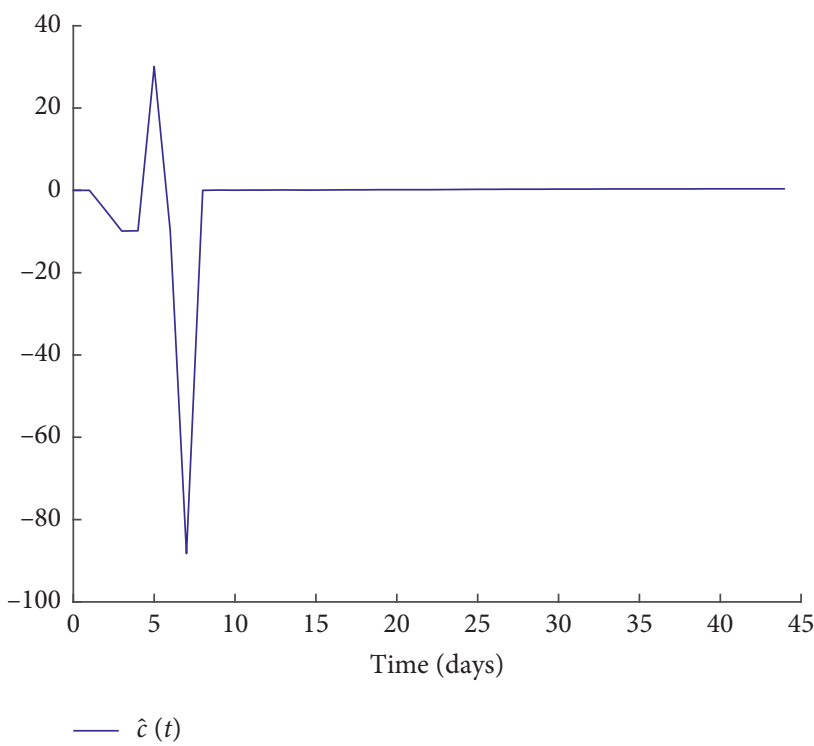

FIGURE 16: Estimation of the carrying capacity from reported data (Italy) and lsqcurvefit Matlab function.

The simulation starts on February 26, 2020, and spans for 200 days. Moreover, Figure 2 displays the number of active cases per day obtained from the SIR model along with the reported number of cases obtained from [53]. As it is proved in Theorem 1 , the solution of the SIR model remains nonnegative and bounded for all time given nonnegative initial conditions. The evolution of the number of active cases per day (the state $I$ ) can be equivalently described by the time-varying (TV) logistic equation (13). Thus, Figure 3 displays the evolution of the state $I$ obtained from the SIR model and the solution to the timevarying equation (13). It is concluded from Figure 3 that both systems are equivalent so that the ad hoc time-varying logistic equation is a single equation that appropriately describes the number of active cases per day. Furthermore, Figures 4 and 5 


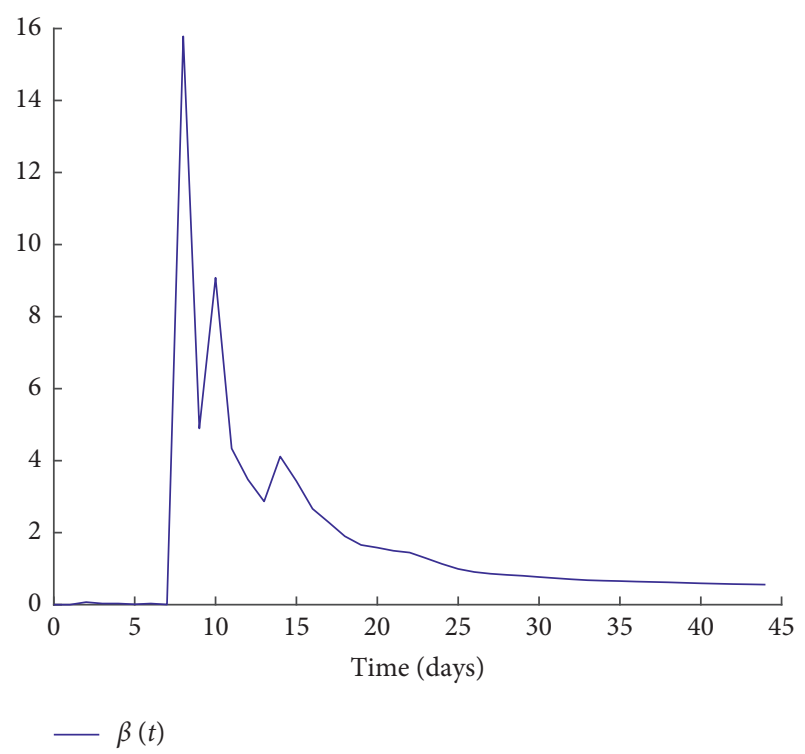

Figure 17: Estimation of $\beta$ from reported data (Italy) and lsqcurvefit Matlab function.

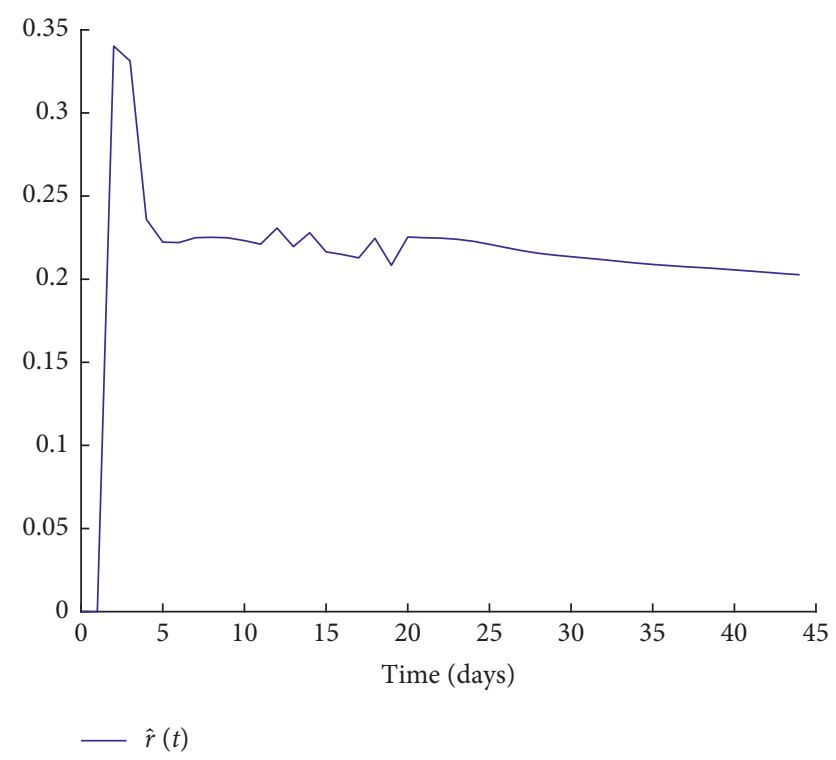

FIGURE 18: Estimation of the Malthusian parameter from reported filtered data (Italy) and lsqcurvefit Matlab function.

display the values of the Malthusian function, $r(t)$, and carrying capacity, $c(t)$, defined in (14), which parameterize the timevarying logistic equation.

Under certain conditions, the time-varying logistic equation can be approximated by a nominal time invariant one that simplifies the model of infection spreading. The main condition for this to hold is the excess $(N(t)-R(t))$ being constant and equal to $(N(0)-R(0))$ along time. Figure 6 shows the evolution of $(N(t)-R(t))$ with time. It is observed in Figure 6 that only during the first days of spreading this condition holds so that the nominal logistic equation is an appropriate model only while the incubation period has not supplied with individuals yet the

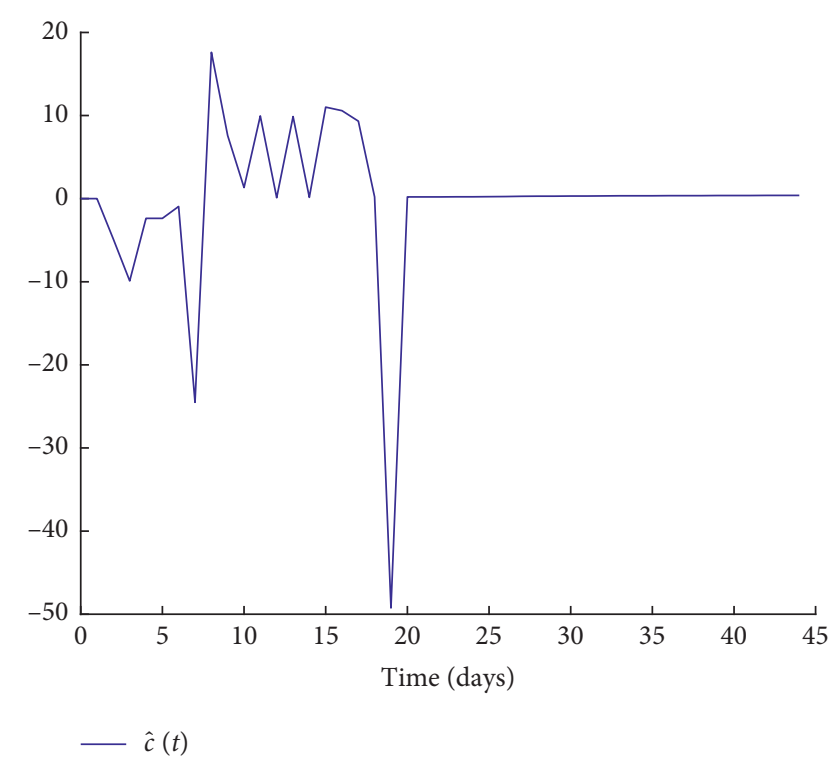

FIGURE 19: Estimation of the carrying capacity from reported filtered data (Italy) and lsqcurvefit Matlab function.

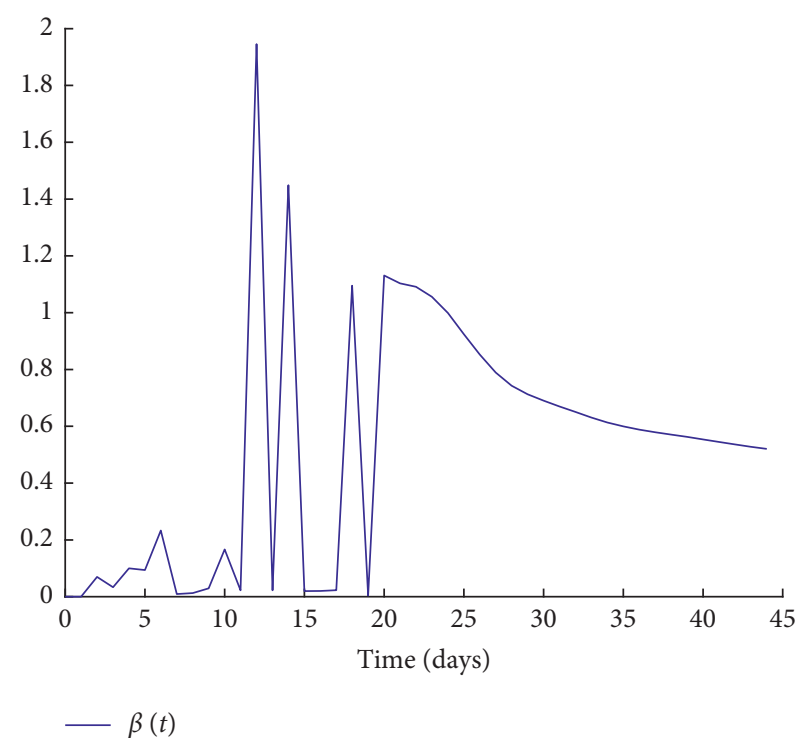

FIgURe 20: Estimation of $\beta$ from reported filtered data (Italy) and lsqcurvefit Matlab function.

subpopulation of immune. Moreover, Figure 7 supports this conclusion where the plots of the SIR model and the timevarying and the nominal logistic equations are displayed. It is observed in Figure 7 that the nominal logistic equation is close to the solution of the SIR model during the first days of simulation. Therefore, the nominal logistic equation may be used as a simplified model to describe the spread of an infection during the first stages, while the time-varying one may be used to describe the spreading for a longer period. It can also be concluded from Figure 6 that $N(t)-R(t) \leq N(0)-R(0)$ for all time since the condition in Proposition 1 trivially holds in this case, where the infectious are always nonnegative and $\rho=\mu=\nu=0$. 


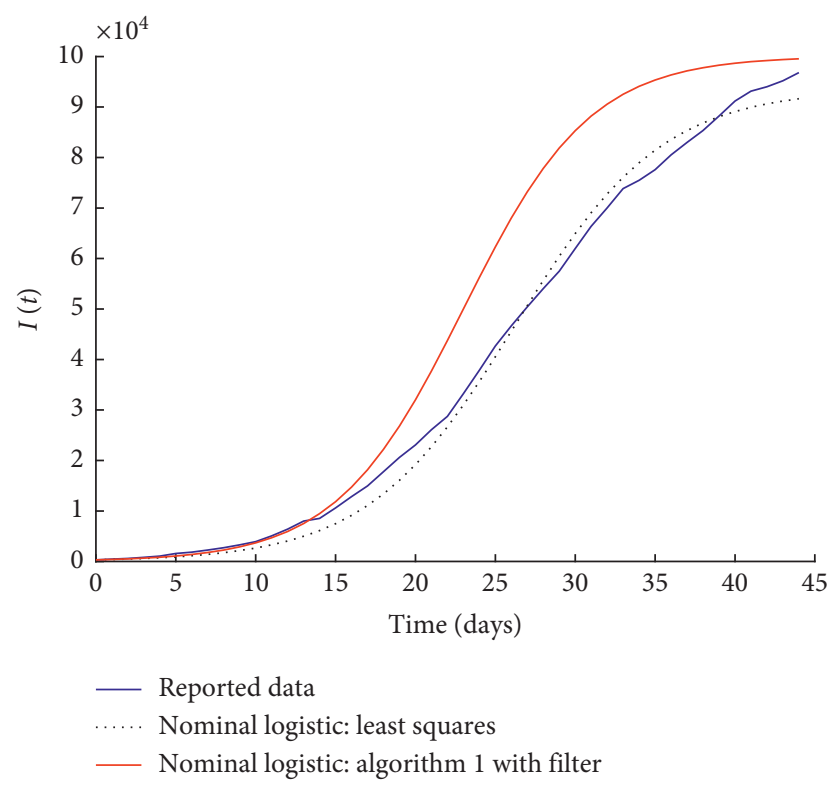

FIGURE 21: Comparison of reported data with the output of the estimated nominal logistic equation.

As a consequence, it is of interest to have an algorithm to estimate the values of the Malthusian and carrying capacity from data in order to obtain a description of the infection spreading during the first stages. Therefore, the algorithm discussed in Section 4 will be applied now to estimate these parameters during the first days of spreading. Initially, the algorithm is applied to the numerical data obtained by simulation from the original SIR model. Thus, consider a constant sampling time of $T_{M}=T_{m}=T_{i}=1$ hour, which is a low sampling time for epidemic systems and the use of equations (A.5)-(A.11) from the Appendix to estimate the values of the carrying capacity and Malthusian parameter. Since the approximation to the nominal logistic equation only holds during the first days of simulation, the first 45 days will only be considered. Figures 8 and 9 display the evolution of the actual and estimated Malthusian parameter and actual and estimated carrying capacity, respectively. It is concluded from Figures 8 and 9 that the estimated Malthusian parameter is close to the actual value, while the carrying capacity is estimated with an error of $20 \%$. The estimated value of $\beta$ is therefore 0.2246 days $^{-1}$, in comparison to the actual one of 0.18 days $^{-1}$. The algorithm provides an estimation of the transmission rate by measuring only the number of active cases in three consecutive time samples. Now, the method will be applied to reported data from Italy, where data are collected every day, i.e., $T_{M}=T_{m}=T_{i}=1$ day.

Figures 10 and 11 show the estimated values of the Malthusian parameter and carrying capacity when the method is applied to reported data from Italy. It can be deduced from these figures that raw data contain too much variability to perform the estimation directly. Therefore, an averaging filter is employed to smoothen the data prior to applying the estimation procedure. Thus, we use the filter $y_{k f}=(1 / 5) \sum_{i=0}^{4} y_{k-i}$, which is the mean value of the last 5 samples. The estimation with filtered values provides
Figures 12 and 13. Moreover, the estimated value of $\beta=|r / c|$ is depicted in Figure 14. From these figures, it can be concluded that the filter smoothens the output of the estimation, but the filtered data still have too much variability to extract the value of $\beta$ as the mean value of the series. The value of $\beta$ at the last stage of simulation (which is the most stable one) is $\beta=0.412$.

Furthermore, a least squares method is now applied to approximate the reported data to a nominal logistic equation. To this end, the lsqcurvefit Matlab function will be used. In this way, the fitting procedure is repeated every time a new data is collected. Figures 15-17 display the evolution of the estimated parameters with time when the least squares method is employed.

The value at the end of simulation is $\beta=0.558$. A filtered version of the data is also used to perform the estimation. In this way, the same filter as before is used to smoothen the data prior to the application of the least squares method. Thus, Figures 18-20 display the evolution of estimated parameters when filtered data are used.

The value of $\beta=0.5204$ is obtained at the end of simulation. Finally, Figure 21 depicts the reported data along with the plot of a nominal logistic equation parameterized by the values obtained at the end of least squares estimation and a nominal logistic equations parameterized by the values obtained at the end of Algorithm 1 execution with filtered data. It is observed in Figure 21 that the least-squares estimated nominal logistic equation is close to the reported data while the nominal equation parametrized by Algorithm 1 is slightly further. Overall, the nominal logistic equation is an appropriate model to describe the spread of an infection during its first stages. The estimation of logistic equation parameters allows obtaining an estimation of the transmission rate of the disease, which provides useful information for decision makers. Algorithm 1 provides an estimation of the 
transmission rate with less computational burden than the least-squares algorithm. However, the treatment of raw data may require the application of denoising or filtering procedures in order data to be smoothen before processing, which is a future research line It is of interest to extend the obtained modeling results with use of vaccination controls [54] and to evaluate results with future evolution of the pandemic under vaccination data.

\section{Conclusions}

This paper has compared an SIR epidemic model with recruitment, demography, and disease-related mortality with a parallel description through an "ad hoc" logistic equation, which is time varying. The logistic equation version of the model allows easily to interpret it via the Malthusian parameter (related to the exponential rate of variation of the solution), the carrying capacity (related to the maximum values which are reached), and the transmission rate, whose value is related to the absolute value of the quotient of the absolute value of the Malthusian parameters and the carrying capacity. To address the process of picking up the data registration in order to estimate the above parameters, an adaptive sampling law is proposed which updates the discrete data accordingly to their rate of variation leading to an optional nonuniform disposal of samples to calculate the, in general, sample-dependent Malthusian parameter and carrying capacity. The transmission rate is then calculated from the above ones and can be averaged on the various intervention phases of quarantine interventions, interventions absence, and programmed de-escalated decisions. Numerical examples which illustrate the methodology are given and discussed based on COVID-19 parameterizations.

\section{Appendix}

\section{A Calculations Related to Step 3 of Algorithm 1}

Take two consecutive updated estimates $\widehat{t}_{i+2}$ of the sampling instant $t_{i+2}$, once the current sampling instant and sampling period $t_{i}$ and $T_{i}$ are known so that $t_{i+1}=t_{i}+T_{i}$ is known:

$$
\widehat{t}_{i+2}=t_{i+1}+T_{i}=t_{i}+2 T_{i} .
$$

Then, rewrite (58) for $t_{i+1}$ and $\widehat{t}_{i+2}$ such that one-step ahead and two-step ahead estimates of $K\left(t_{i}\right)$ and $r\left(t_{i}\right)$ are calculated "a posteriori" after $\widehat{t}_{i+2}$ has occurred as follows from the registered measures $I\left(t_{i}\right), I\left(t_{i+1}\right)=I\left(t_{i}+T_{i}\right)$, and $I\left(\widehat{t}_{i+2}\right)=I\left(t_{i+1}+T_{i}\right)=I\left(t_{i}+2 T_{i}\right)$ :

$$
\begin{aligned}
\widehat{K}\left(t_{i}, t_{i+1}\right) & =\frac{I\left(t_{i+1}\right) I\left(t_{i}\right)\left(1-e^{-T_{i} \widehat{r}\left(t_{i}, t_{i+1}\right)}\right)}{I\left(t_{i}\right)-I\left(t_{i+1}\right) e^{-T_{i} \widehat{r}\left(t_{i}, t_{i+1}\right)}}, \\
\widehat{K}\left(t_{i}, \widehat{t}_{i+2}\right) & =\frac{I\left(\widehat{t}_{i+2}\right) I\left(t_{i+1}\right)\left(1-e^{-T_{i} \widehat{r}\left(t_{i}, \bar{t}_{i+2}\right)}\right)}{I\left(t_{i+1}\right)-I\left(\widehat{t}_{i+2}\right) e^{-T_{i} \widehat{r}\left(t_{i}, \hat{t}_{i+2}\right)}} .
\end{aligned}
$$

Then, the above equations are calculated under the constraints:

$$
\begin{aligned}
K\left(t_{i}\right) & =\widehat{K}\left(t_{i}, t_{i+1}\right)=\widehat{K}\left(t_{i}, \widehat{t}_{i+2}\right), \\
r\left(t_{i}\right) & =\widehat{r}\left(t_{i}, t_{i+1}\right)=\widehat{r}\left(t_{i}, \widehat{t}_{i+2}\right),
\end{aligned}
$$

which lead to

$$
\begin{aligned}
K\left(t_{i}\right) & =\frac{I\left(t_{i}+T_{i}\right) I\left(t_{i}\right)\left(1-e^{-T_{i} r\left(t_{i}\right)}\right)}{I\left(t_{i}\right)-I\left(t_{i}+T_{i}\right) e^{-T_{i} r\left(t_{i}\right)}} \\
& =\frac{I\left(t_{i}+2 T_{i}\right) I\left(t_{i}+T_{i}\right)\left(1-e^{-T_{i} r\left(t_{i}\right)}\right)}{I\left(t_{i}+T_{i}\right)-I\left(t_{i}+2 T_{i}\right) e^{-T_{i} r\left(t_{i}\right)}}
\end{aligned}
$$

so that

$$
\frac{I\left(t_{i}+T_{i}\right) I\left(t_{i}\right)}{I\left(t_{i}\right)-I\left(t_{i}+T_{i}\right) e^{-T_{i} r\left(t_{i}\right)}} \times \frac{I\left(t_{i}+T_{i}\right)-I\left(t_{i}+2 T_{i}\right) e^{-T_{i} r\left(t_{i}\right)}}{I\left(t_{i}+2 T_{i}\right) I\left(t_{i}+T_{i}\right)}=1
$$

so that

$$
a_{i} e^{-2 T_{i} r\left(t_{i}\right)}-b_{i} e^{-T_{i} r\left(t_{i}\right)}+c_{i}=0,
$$

whose roots are

$$
e^{-T_{i} r\left(t_{i}\right)}=\frac{b_{i} \pm \sqrt{b_{i}^{2}-4 a_{i} c_{i}}}{2 a_{i}}
$$

where

$$
\begin{aligned}
& a_{i}=I\left(t_{i}+T_{i}\right) I\left(t_{i}+2 T_{i}\right), \\
& b_{i}=I\left(t_{i}+T_{i}\right)^{2}+I\left(t_{i}\right) I\left(t_{i}+2 T_{i}\right), \\
& c_{i}=I\left(t_{i}\right) I\left(t_{i}+T_{i}\right),
\end{aligned}
$$

leading to

$$
b_{i}^{2}-4 a_{i} c_{i}=\left(I\left(t_{i}+T_{i}\right)-I\left(t_{i}\right) I\left(t_{i}+2 T_{i}\right)\right)^{2} \geq 0
$$

so that the roots of (A.6) are real. The positive root leads to the Malthusian parameter at $t_{i}$ :

$$
r\left(t_{i}\right)=\frac{1}{T_{i}} \ln \frac{2 a_{i}}{b_{i}+\sqrt{b_{i}^{2}-4 a_{i} c_{i}}}
$$

and then the carrying capacity is calculated from (A.5).

Remark A.1. If past measures of the infection are used instead of either a prediction or a selection of provided registered values after the current time instant $t_{i}$, one uses the subsequent equations: 


$$
\begin{aligned}
& \widehat{c}\left(t_{i}\right)=\widehat{K}\left(t_{i}\right)=\frac{I\left(t_{i}\right) I\left(t_{i-1}\right)\left(1-e^{-T_{i-2} \widehat{r}\left(t_{i-2}\right)}\right)}{I\left(t_{i-1}\right)-I\left(t_{i}\right) e^{-T_{i-2} \widehat{r}\left(t_{i-1}\right)},}, \\
& \widehat{c}\left(t_{i-1}\right)=\widehat{K}\left(t_{i-1}\right)=\frac{I\left(t_{i-1}\right) I\left(t_{i-2}\right)\left(1-e^{-T_{i-2} \widehat{r}\left(t_{i-2}\right)}\right)}{I\left(t_{i-2}\right)-I\left(t_{i-1}\right) e^{-T_{i-2} \widehat{r}\left(t_{i-2}\right)}},
\end{aligned}
$$

and equalizing $\widehat{K}\left(t_{i}\right)=\widehat{K}\left(t_{i-1}\right)$ and $T_{i-1}=T_{i-2}$ one gets the following alternative solution to (A.7)-(A.12):

$$
e^{-T_{i-1} \widehat{r}\left(t_{i-1}\right)}=\frac{I\left(t_{i-2}\right)\left(I\left(t_{i}\right)-I\left(t_{i-1}\right)\right)}{I\left(t_{i}\right)\left(I\left(t_{i-1}\right)-I\left(t_{i-2}\right)\right)} .
$$

\section{Data Availability}

There are datasets previously available at https://www. worldometers.info/coronavirus/, which have been used in the numerical simulations. These prior studies and datasets are cited at relevant places within the text as reference [53].

\section{Conflicts of Interest}

The authors declare that they have no conflicts of interest regarding the publication of this manuscript.

\section{Acknowledgments}

The authors are grateful to the Spanish Government for Grant RTI2018-094336-B-I00 (MCIU/AEI/FEDER, UE) and to the Basque Government for Grant IT1207-19. The also thank the Spanish Institute of Health Carlos III for its support through Grant COV 20/01213. Finally, they thank the referees for their useful suggestions.

\section{References}

[1] L. Rass and J. Radcliffe, Spatial Deterministic Epidemics, Mathematical Surveys and Monographs, American Mathematical Society, Providence, RI, USA, 2003.

[2] M. J. Keeling and P. Rohani, Modeling Infectious Diseases, Princeton University Press, Princeton, NJ, USA, 2008.

[3] Z. S. Shuai and P. Van der Driessche, "Global stability of infectious disease models using Lyapunov functions," SIAM Journal on Applied Mathematics, vol. 73, no. 4, pp. 1513-1532, 2013.

[4] M. De la Sen, R. Nistal, S. Alonso-Quesada, and A. Ibeas, "Some formal results on positivity, stability, and endemic steady-state attainability based on linear algebraic tools for a class of epidemic models with eventual incommensurate delays," Discrete Dynamics in Nature and Society, vol. 2019, Article ID 8959681, 22 pages, 2019.

[5] T. Harko, F. S. N. Lobo, and M. K. Mak, "Exact analytical solutions of the Susceptible-Infected-Recovered (SIR) epidemic model and of the SIR model with equal death and birth rates," Applied Mathematics and Computation, vol. 236, pp. 184-194, 2014.

[6] H. W. Hethcote, "The mathematics of infectious diseases," SIAM Review, vol. 42, no. 4, pp. 599-653, 2000.
[7] R. Verma, V. K. Sehgal, and V. Nitin, "Computational stochastic modelling to handle the crisis occurred during community epidemic," Annals of Data Science, vol. 3, no. 2, pp. 119-133, 2016.

[8] A. Iggidr and M. O. Souza, "State estimators for some epidemiological systems," Journal of Mathematical Biology, vol. 78, no. 1-2, pp. 225-256, 2019.

[9] A. Kumar, K. Goel, and Nilam, "A deterministic time-delayed SIR epidemic model: mathematical modeling and analysis," Theory in Biosciences, vol. 139, no. 1, pp. 67-76, 2020.

[10] M. L. Taylor and T. W. Carr, "An SIR epidemic model with partial temporary immunity modeled with delay," Journal of Mathematical Biology, vol. 59, no. 6, pp. 841-880, 2009.

[11] L. Bai, J. J. Nieto, and J. M. Uzal, "On a delayed epidemic model with non-instantaneous impulses," Communications in Pure and Applied Analysis, vol. 19, no. 4, pp. 1915-1930, 2020.

[12] C. Conell McCluskey, "Global stability of an SIR epidemic model with delay and general nonlinear incidence," Mathematical Biosciences and Engineering, vol. 7, no. 4, pp. 837-850, 2010.

[13] M. De la Sen, A. Ibeas, S. Alonso-Quesada, and R. Nistal, "On a SIR model in a patchy environment under constant and feedback decentralized controls with asymmetric parameterizations," Symmetry-Basel, vol. 11, no. 3, p. 430, 2019.

[14] S. B. Cui and M. Bai, "Mathematical analysis of population migration and its effects to spread of epidemics," Discrete and Continuous Dynamical Systems-Series B, vol. 20, no. 9, pp. 2819-2858, 2015.

[15] M. De la Sen, S. Alonso-Quesada, A. Ibeas, and R. Nistal, "On an SEIADR epidemic model with vaccination, treatment and dead-infectious corpses removal controls," Mathematics and Computers in Simulation, vol. 163, pp. 47-79, 2019.

[16] I. Al-Darabsah and Y. Yuan, "A time-delayed epidemic model for Ebola disease transmission," Applied Mathematics and Computation, vol. 290, pp. 307-325, 2016.

[17] Z. L. He and L. F. Nie, "The effect of pulse vaccination and treatment on SIR epidemic model with media impact," Discrete Dynamics in Nature and Society, vol. 2015, Article ID 532494, 12 pages, 2015.

[18] J. Hou and Z. D. Teng, "Continuous and impulsive vaccination of SEIR epidemic models with saturation incidence rates," Mathematics and Computers in Simulation, vol. 79, no. 10, pp. 3038-3054, 2009.

[19] A. Boonyaprapasorn, N. Natsupakpong, P. S. Ngiamsunthorn, and K. Thung-Od, "An application of finite time synergetic control for vaccination in epidemic systems," in Proceedings of the 2017 IEEE Conference on Systems, Process and Control (ICSPC), pp. 30-25, Melaka, Malaysia, December 2017.

[20] A. Boonyaprapasorn, N. Natsupakpong, P. S. Ngiamsunthorn, and $\mathrm{K}$. Thung-Od, "Fractional order sliding mode control for vaccination in epidemic systems," in Proceedings of the 2017 2nd International Conference on Control and Robotics Engineering (ICCRE 2017), pp. 30-25, Bangkok, Thailand, April 2017.

[21] T. Sethaput and A. Boonyaprapasorn, "Fractional order sliding mode control applying on the HIV infection system," in Proceedings of the 2018 International Conference on Artificial Life and Robotics ICAROB 2018, pp. 157-160, Beppu, Japan, February 2018.

[22] A. Ibeas, M. De la Sen, and S. Alonso-Quesada, "Robust sliding control of SEIR epidemic models," Mathematical Problems in Engineering, vol. 2014, Article ID 104764, 11 pages, 2014. 
[23] O. Zakary, A. Larrache, M. Rachik, and I. Elmouki, "Effect of awareness programs and travel-blocking operations in the control of HIV/AIDS outbreaks: a multi-domains SIR model," Advances in Difference Equations, vol. 2016, p. 169, 2016.

[24] S. Bidah, O. Zakary, and M. Rachik, "Stability and global sensitivity analysis for an agree-disagree model: partial rank correlation coefficient and Latin hypercube sampling methods," International Journal of Differential Equations, vol. 2020, Article ID 5051248, 14 pages, 2020.

[25] Z. Chen, "Discrete-time vs. Continuous-time epidemic models in networks," IEEE Access, vol. 7, pp. 127669-127677, 2019.

[26] H. Boutayeb, S. Bidah, O. Zakary, and M. Rachik, "A new simple epidemic discrete-time model describing the dissemination of information with optimal control strategy," Discrete Dynamics in Nature and Society, vol. 2020, Article ID 7465761, 11 pages, 2020.

[27] A. D’Onofrio, “Mixed pulse vaccination strategy in epidemic model with realistically distributed infectious and latent times," Applied Mathematics and Computation, vol. 151, no. 1, pp. 181-187, 2004.

[28] I. Ameen, D. Baleanu, and H. M. Ali, “An efficient algorithm for solving the fractional optimal control of SIRV epidemic model with a combination of vaccination and treatment," Chaos, Solitons \& Fractals, vol. 137, Article ID 109892, 2020.

[29] M. De la Sen, R. Nistal, A. Ibeas, and A. J. Garrido, "On the use of entropy issues to evaluate and control the transients in some epidemic models," Entropy, vol. 22, no. 5, p. 534, 2020.

[30] M. De la Sen, A. Ibeas, and R. Nistal, "On the entropy of events under eventually inflated or deflated probability constraints. Application to the supervision of epidemic models under vaccination controls," Entropy, vol. 22, no. 3, p. 284, 2020.

[31] W. B. Wang, Z. N. Wu, C. F. Wang, and R. F. Hu, "Modelling the spreading rate of controlled communicable epidemics through and entropy-based thermodynamic model," Science China Physics, Mechanics and Astronomy, vol. 56, no. 11, pp. 2143-2150, 2013.

[32] M. Koivu-Jolma and A. Annila, "Epidemic as a natural process," Mathematical Biosciences, vol. 299, pp. 97-102, 2018.

[33] C. Yang and J. Wang, "A mathematical model for the novel coronavirus epidemic in Wuhan, China," AIMS Mathematical Biosciences and Engineering, vol. 17, no. 3, pp. 2708-2724, 2020.

[34] H. F. Huo and Z. P. Ma, "Dynamics of a delayed epidemic model with non-monotonic incidence rate," Communications in Nonlinear Science and Numerical Simulation, vol. 15, no. 4, pp. 459-468, 2010.

[35] X. Li, W. Y. Liu, C. L. Zhao, X. Zhang, and D. Y. Yi, "Locating multiple sources of contagion in complex networks under the SIR model," Applied Sciences-Basel, vol. 9, no. 20, p. 4472, 2019.

[36] M. H. Yang and A. R. R. Freitas, "Biological view of vaccination described by mathematical modellings: from rubella to dengue vaccines," Mathematical Biosciences and Engineering: $M B E$, vol. 16, no. 4, pp. 3195-3214, 2019.

[37] P. R. Thakare and S. S. Mathurkar, "Modeling of epidemic spread by social interactions," in Proceedings of the 2016 IEEE International Conference on Recent Trends in Electronics, Information \& Communication Technology (RTEICT), pp. 1320-1324, Bangalore, India, May 2016.

[38] F. Darabi Sahneh and C. Scoglio, "Epidemic spread in human networks," in Proceedings of the 50th IEEE Conference on Decision and Control and European Control Conference, pp. 3008-3013, Orlando, FL, USA, December 2011.
[39] Z. Zhang and H. Wang, "Epidemic source tracing on social contact networks," in Proceedings of the 2015 17th International Conference on E-Health Networking, Application \& Services (HealthCom), pp. 354-357, Boston, MA, USA, October 2015.

[40] W.-T. Zha, F.-R. Pang, N. Zhou et al., "Research about the optimal strategies for prevention and control of varicella outbreak in a school in a central city of China: based on an SEIR dynamic model," Epidemiology and Infection, vol. 148, p. e56, 2020.

[41] K. Y. Ng and M. M. Gui, "COVID-19: development of a robust mathematical model and simulation package with consideration for ageing population and time delay for control action and resusceptibility," Physica (D): Nonlinear Phenomena, vol. 411, 2020 In press, Article ID 132599.

[42] R. K. Kumar, M. Rani, A. S. Bhagavathula et al., "Prediction of the COVID-19 pandemic for the top 15 affected countries: advanced autoregressive integrated moving average (ARIMA) model," JMIR Public Health and Surveillance, vol. 6, no. 2, Article ID e19115, 2020.

[43] T. Kuniya and H. Inaba, "Possible effects of mixed prevention strategy for COVID-19 epidemic: massive testing, quarantine and social distance," AIMS Public Health, vol. 7, no. 3, pp. 490-503. In press, 2020.

[44] Y. Liu, "Death toll estimation for COVID-19: is the curve flattened yet?" https://ssrn.com/abstract $=3592343$.

[45] Mortality Rate of COVID-19 in Spain as of May 22, 2020, by age group, https://www.statista.com/statistics/1105596/covid19-mortality-rate-by-age-group-in-spain-march/Access\% 20date, 2020.

[46] I. K. Abdulrahman, "SimCOVID: an open source simulation program for the COVID-19 outbreak," 2020, https://www. medrxiv.org/content/10.1101/2020.04.13.20063354v2 medRxivpreprint, Paper in Collection COVID-19 SARS-CoV-2 Preprints from medRxiv and bioRxiv 2020.

[47] N. Fabiano and S. Radenović, "On COVID-19 diffusion in Italy: data analysis and possible outcome," Vojnotehnicki Glasnik, vol. 68, no. 2, pp. 216-224, 2020.

[48] M. De La Sen, "Adaptive sampling for improving the adaptation transients in hybrid adaptive control," International Journal of Control, vol. 41, no. 5, pp. 1189-1205, 1985.

[49] M. Delasen, "A method for improving the adaptation transient using adaptive sampling," International Journal of Control, vol. 40, no. 4, pp. 639-665, 1984.

[50] R. Dorf, M. Phillips, and M. C. Farren, "Adaptive sampling frequency for sampled-data control systems," IRE Transactions on Automatic Control, vol. 7, no. 1, pp. 38-47, 1962.

[51] T. C. Hsia, "Analytic design of adaptive sampling control law in sampled-data systems," IEEE Transactions on Automatic Control, vol. AC19, no. 1, pp. 39-42, 1974.

[52] I. Cooper, A. Mondal, and C. G. Antonopoulos, "A SIR model assumption for the spread of COVID-19 in different communities," Chaos, Solitons And Fractals, vol. 139. 2020 In press, Article ID 110057.

[53] Worldometer, https://www.worldometers.info/coronavirus/ Acces\%20date\%20July, 2020.

[54] M. De la Sen, R. P. Agarwal, A. Ibeas, and S. Alonso-Quesada, "On a generalized time-varying SEIR epidemic model with mixed point and distributed time-varying delays and combined regular and impulsive vaccination controls," Advances in Difference Equations, vol. 2010, Article ID 281612, 42 pages, 2010. 